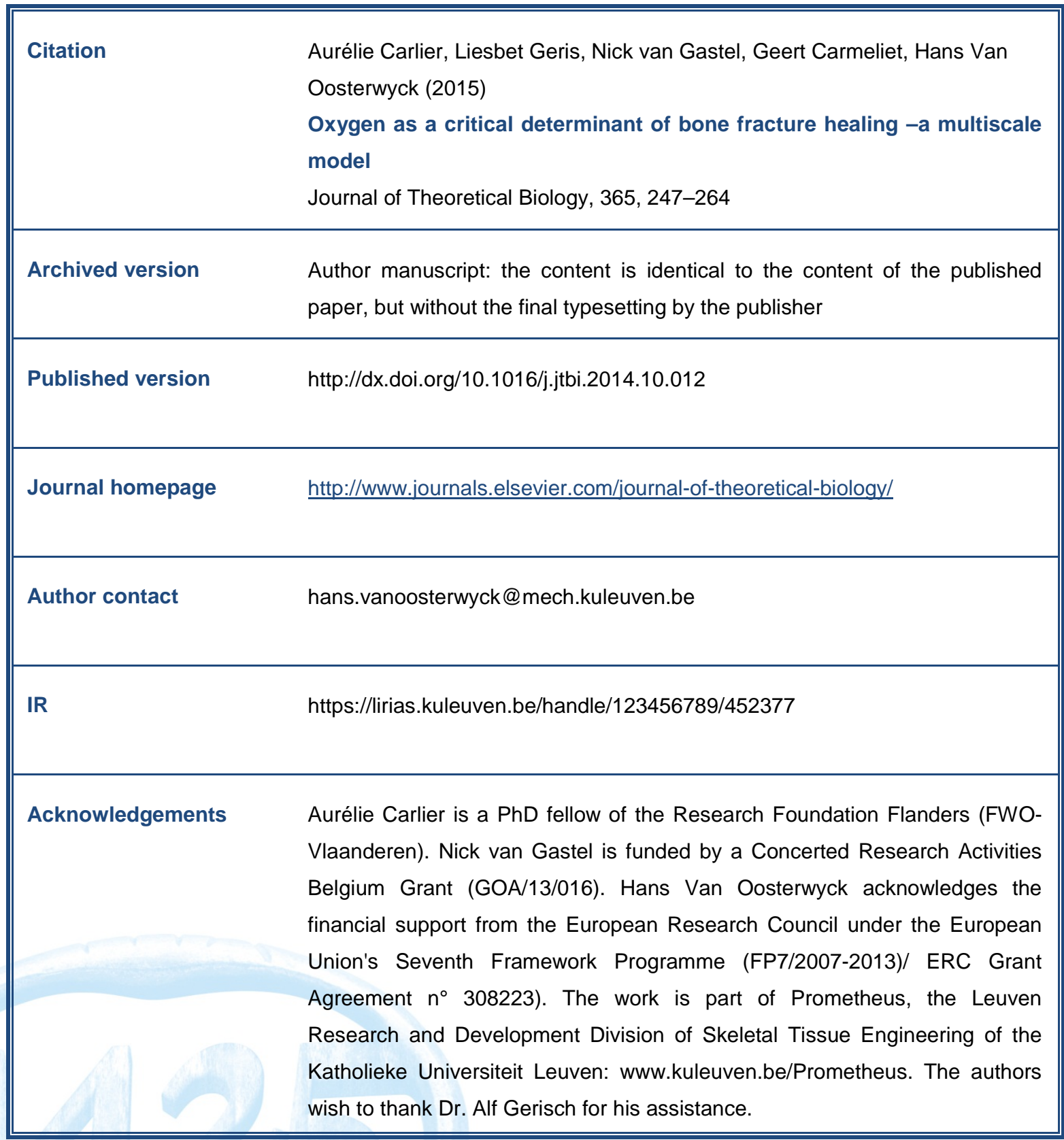




\section{Oxygen as a critical determinant of bone fracture healing - a multiscale model}

Aurélie Carlier ${ }^{1,2,4}$, Liesbet Geris ${ }^{1,2,4}$, Nick van Gastel ${ }^{3,4}$, Geert Carmeliet ${ }^{3,4}$, Hans Van Oosterwyck ${ }^{1,4}$

Affiliation:

${ }^{1}$ Biomechanics Section, KU Leuven, Celestijnenlaan 300 C, PB 2419, 3000 Leuven, Belgium.

${ }^{2}$ Biomechanics Research Unit, University of Liege, Chemin des Chevreuils 1 - BAT 52/3, 4000 Liege 1, Belgium.

${ }^{3}$ Clinical and Experimental Endocrinology, KU Leuven, O\&N 1, Herestraat 49, PB 902, 3000 Leuven, Belgium.

${ }^{4}$ Prometheus, Division of Skeletal Tissue Engineering, KU Leuven, O\&N 1, Herestraat 49, PB 813, 3000 Leuven, Belgium.

\section{Corresponding author:}

Hans Van Oosterwyck

Biomechanics Section

Celestijnenlaan 300C, bus 2419

3000 Leuven, Belgium

tel +3216327067

fax +3216 327994

E-mail: hans.vanoosterwyck@mech.kuleuven.be 


\section{Highlights}

- The influence of oxygen was incorporated in a multiscale model of fracture healing.

- The results of the oxygen model were compared with experimental observations.

- An extensive sensitivity analysis of the oxygen model indicated its robustness.

- Adequate spatiotemporal oxygen patterns appear to be critical for bone healing.

\section{Keywords}

oxygen - angiogenesis - fracture healing - multiscale model - non-union

\section{Abstract}

A timely restoration of the ruptured blood vessel network in order to deliver oxygen and nutrients to the fracture zone is crucial for successful bone healing. Indeed, oxygen plays a key role in the aerobic metabolism of cells, in the activity of a myriad of enzymes as well as in the regulation of several (angiogenic) genes. In this paper, a previously developed model of bone fracture healing is further improved with a detailed description of the influence of oxygen on various cellular processes that occur during bone fracture healing. Oxygen ranges of the cell-specific oxygen-dependent processes were established based on the state-of-the art experimental knowledge through a rigorous literature study. The newly developed oxygen model is compared with previously published experimental and in silico results. An extensive sensitivity analysis was also performed on the newly introduced oxygen thresholds, indicating the robustness of the oxygen model. Finally, the oxygen model was applied to the challenging clinical case of a critical sized defect $(3 \mathrm{~mm})$ where it predicted the formation of a fracture non-union. Further model analyses showed that the harsh hypoxic conditions in the central region of the callus resulted in cell death and disrupted bone healing thereby indicating the importance of a timely vascularization for the successful healing of a large bone defect. In conclusion, this work demonstrates that the oxygen model 
is a powerful tool to further unravel the complex spatiotemporal interplay of oxygen delivery, diffusion and consumption with the several healing steps, each occurring at distinct, optimal oxygen tensions during the bone repair process.

\section{Introduction}

\subsection{Normal fracture healing}

When a bone fractures, the bone architecture distorts and blood vessels rupture, thereby filling the fracture site with blood which rapidly coagulates to form a blood clot or hematoma (Murao et al., 2013). Since the damaged vasculature fails to provide sufficient oxygen and nutrients, the injury site gradually becomes hypoxic and the surrounding tissues start to degrade (Cameron et al., 2013). This triggers the invasion of inflammatory cells, macrophages and leukocytes, marking the start of the inflammatory phase. Simultaneously, growth factors and cytokines produced by the cells in the hematoma and surrounding tissues attract fibroblasts, mesenchymal stem cells (MSCs) and endothelial cells to the trauma site (Taguchi et al., 2005). The fracture callus fills with granulation tissue, forming the soft callus, in which the MSCs start to differentiate. In the periosteal region, near the bone cortex where oxygen is available, the MSCs differentiate directly towards osteogenic cells. These newly formed osteoblasts produce a woven bone matrix (intramembranous ossification). In the hypoxic central fracture area, the mesenchymal stem cells will first differentiate into chondrocytes which produce a cartilaginous template that mechanically stabilizes the fracture zone. The hard callus formation stage starts with the invasion of blood vessels into this cartilaginous template. The new-sprung vasculature brings along osteoblasts that produce a hard tissue callus of mineralized woven bone matrix (endochondral bone 
formation). When the bony callus bridges the fracture gap, a clinical union is reached. In the final remodeling phase, the hard callus is remodeled by osteoclasts and osteoblasts, gradually replacing the immature woven bone by lamellar bone and returning the bone to its original shape, size and strength (Einhorn, 1998).

\subsection{The role of oxygen in fracture healing}

Oxygen is essential for multiple cellular functions occurring during normal conditions as well as during repair processes like fracture healing. Firstly, it is required for the aerobic metabolism of cells, thereby producing ATP for normal cellular function (Lu et al., 2013a; Maes et al., 2012). Secondly, oxygen is important for the activity of many enzymes (Lu et al., 2013a;Xie et al., 2009). Thirdly, a lack of oxygen induces the expression of several (angiogenic) genes through the hypoxia inducible factor (HIF)-pathway (Pugh and Ratcliffe, 2003; Maes et al., 2012;Wan et al., 2008;Komatsu and Hadjiargyrou, 2004;Bouletreau et al., 2002). Fourthly, through the molecular mechanisms mentioned above, oxygen has a profound effect on the differentiation and proliferation capacity of MSCs, chondrocytes and osteoblasts (Malladi et al., 2006;Xu et al., 2007;Grayson et al., 2007; Lennon et al., 2001; Holzwarth et al., 2010;Wagegg et al., 2012;Zscharnack et al., 2009;Meyer et al., 2010; Hirao et al., 2006; Ren et al., 2006; Merceron et al., 2010). Lennon et al. (Lennon et al., 2001) observed for example that rat MSCs proliferated faster and formed more colonies in low oxygen ( $5 \%$ oxygen tension) than in control conditions ( $20 \%$ oxygen tension). Similar results were obtained by Grayson et al. (Grayson et al., 2007). They report a 30-fold higher expansion of the human MSCs under $2 \%$ oxygen tension with respect to $20 \%$ oxygen tension (Grayson et al., 2007). Finally, it was shown that prolonged hypoxic conditions lead to cell 
death, delayed chondrogenic and osteogenic differentiation and impaired fracture healing (Lu et al., 2013b;Brinker and Bailey, 1997; Lu et al., 2007).

\subsection{Mathematical models of fracture healing including oxygen}

Since oxygen influences many critical processes of fracture healing, as was mentioned in the previous section, mathematical models of fracture healing should consider it explicitly, in this way providing additional opportunities to deepen the scientific understanding of the biological mechanisms at hand. In this section we will give a brief overview of the most recent mathematical models of fracture healing that include oxygen, as this is the focus of this study. For comprehensive reviews on mathematical models of fracture healing, we refer the reader to Geris et al. (Geris et al., 2009), Isaksson et al. (Isaksson, 2012) and Pivonka et al. (Pivonka and Dunstan, 2012).

Simon et al. used fuzzy logic rules to describe the interaction between mechanical stability, revascularization and tissue differentiation during fracture healing with three main variables: vascular perfusion, cartilage concentration and bone concentration (Simon et al., 2011;Chen et al., 2009). They show that both mechanical stabilization as well as sufficient nutrient supply are essential for bone healing since a less stabilized osteotomy leads to slower revascularization and delayed bony bridging. An inadequate nutrient supply, resulting from an increased gap size, would also lead to the formation of a non-union. However, they model the dynamics of endothelial cells as well as the nutrient delivery by diffusion equations with constant diffusion coefficients, i.e. the vessel growth will continue until a uniform density is reached. As such, Chen et al. fail to capture the prolonged absence of healing resulting in a clinical non-union since their model, given enough time, will eventually result in complete bony bridging. 
Burke et al. were able to predict all the major events of fracture repair by defining substrate stiffness and oxygen tension as key regulators of MSC differentiation (Burke and Kelly, 2012). However, they model angiogenesis as a diffusive process, thereby neglecting the discrete nature of the vascular tree (Burke and Kelly, 2012). Moreover, in their model only the differentiation of MSCs is made oxygen dependent (Burke and Kelly, 2012), whereas experimental evidence indicates that multiple cellular processes are regulated by oxygen.

Geris et al. (Geris et al., 2008) developed a model that describes the bone regeneration process as a spatiotemporal variation in density of 12 continuous variables: mesenchymal stem cells, chondrocytes, osteoblasts, fibroblasts, endothelial cells, cartilage matrix, bone matrix, fibrous matrix, vascular matrix, osteogenic growth factors, chondrogenic growth factors and angiogenic growth factors. Peiffer et al. (Peiffer et al., 2011) extended the fracture healing model developed by Geris et al. (Geris et al., 2008) by including a discrete, lattice-free description of endothelial tip cell migration and angiogenesis instead of a continuum description of the vasculature (by means of a vascular density). This modification not only resulted in a more realistic description of angiogenesis, it also allowed to explicitly model oxygen as a variable influencing the fracture healing process through its release from the newly formed vessel network. As such, the model of Peiffer et al. correctly captured the different aspects of bone regeneration as well as some important aspects of angiogenesis like blood vessel growth, branching and anastomosis (Peiffer et al., 2011). The model of Peiffer et al. was further refined by Carlier et al. by introducing an intracellular level in every endothelial cell describing the Dll4-Notch signaling pathway (Carlier et al., 2012) thereby replacing the phenomenological rules of tip cell selection used by Peiffer et al. (Peiffer et al., 2011). Due to its multiscale nature, the so called MOSAIC model (multiscale model of

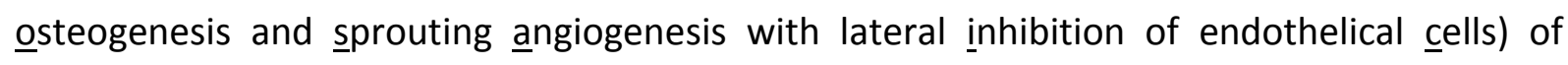


Carlier et al. was able to simulate the bone regeneration process accurately, as well as to reproduce many experimentally observed aspects of tip cell selection: the salt and pepper pattern seen for endothelial cell fates, an increased tip cell density in heterozygous DII4 knockout cases and an excessive number of tip cells in high VEGF (vascular endothelial growth factor) environments.

\subsection{Objectives of this study}

As indicated above, oxygen clearly plays a key role in fracture healing. Indeed, it appears that many biological processes that take place during fracture healing (e.g. proliferation, differentiation, cell death) occur at cell-specific optimal oxygen tensions. Some of the most recent mathematical models of fracture healing have tried to incorporate the role of oxygen in fracture healing, however none of the aforementioned models (including our own) explicitly captures the influence of oxygen on cellular proliferation, differentiation, hypoxia signaling and cell death. We hypothesize that the spatiotemporal distribution of oxygen tension, influenced by amongst others cellular consumption as well as the timely revascularization of the callus, is an important determinant of fracture healing. Therefore, this study will establish a new computational model of fracture healing that is able to more accurately describe the regulatory properties of oxygen on cellular processes occurring during normal and impaired fracture healing. This goal is accomplished by combining the state-of-the-art knowledge on the influence of oxygen on the behavior of skeletal cells with a previously developed multiscale model of bone fracture healing (Carlier et al., 2012). The results of the newly developed oxygen model are compared with experimental data from literature (Harrison et al., 2003). Moreover, an extensive sensitivity analysis is performed on the newly introduced parameters. Finally, the model is applied to critically sized defects in order to explore possible causes of impaired bone healing. 


\section{Materials and Methods}

\subsection{Mathematical model}

\subsubsection{Model overview}

The new oxygen model builds upon a previously published multiscale model of bone fracture healing and consists of (1) a tissue level describing the various key processes of bone fracture healing with 10 continuous variables, (2) a cellular level representing the developing vasculature with discrete endothelial cells and (3) an intracellular level that defines the internal dynamics of the DII4-Notch signaling pathway in every endothelial cell (Carlier et al., 2012). The resulting hybrid framework, which combines PDEs at the tissue level with an agent-based description at the cellular level, is computationally efficient and suitable to answer the research question at hand, i.e. the investigation of the influence of oxygen on bone fracture healing. A schematic overview of the multiscale oxygen model is given in Figure 1. 


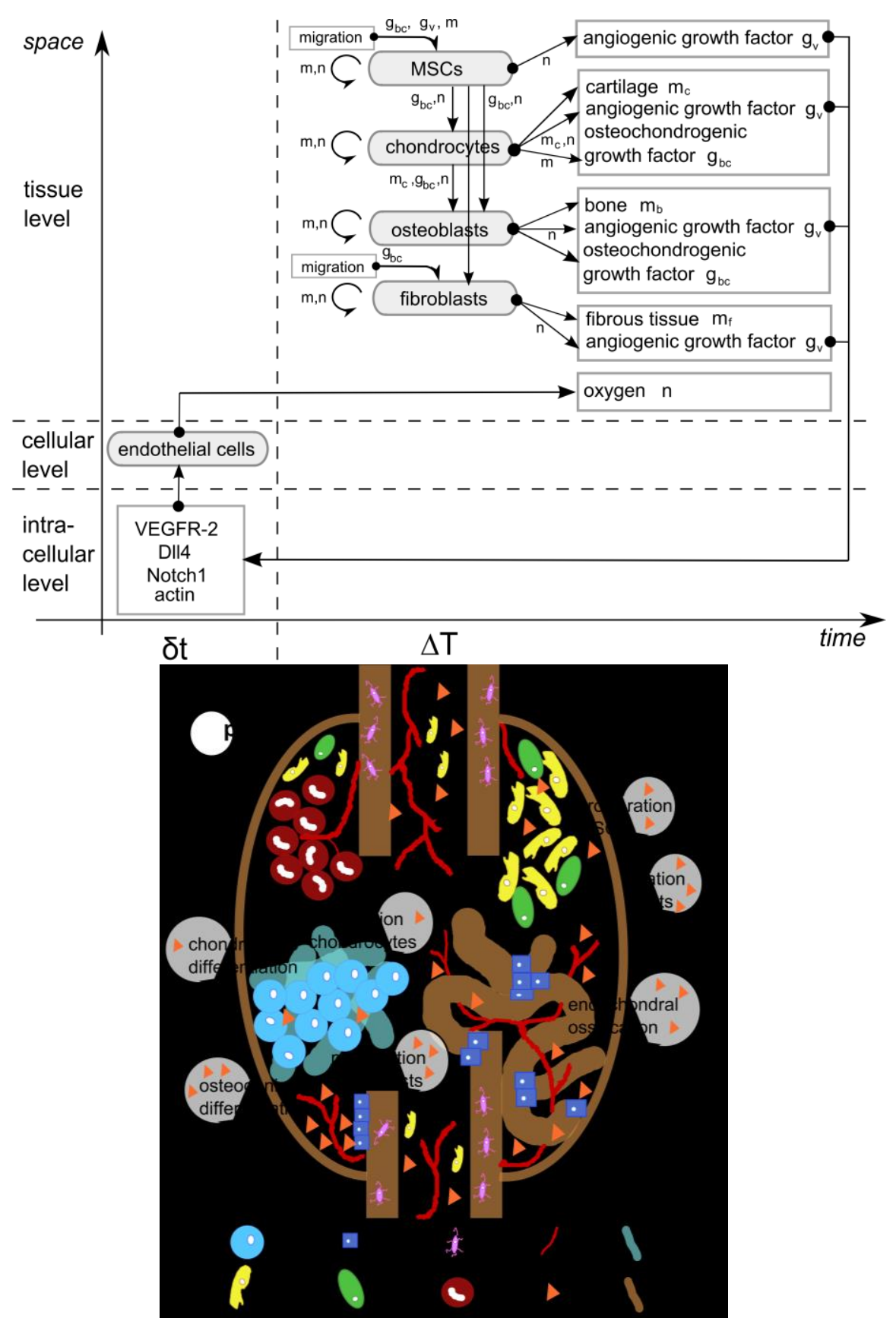

Figure 1: (top) Scale separation map of the multiscale oxygen model indicating schematically the modeled processes at different spatial and temporal scales. The intracellular variables govern the endothelial cell (EC) behavior. At the tissue scale, cells (MSCs, chondrocytes, osteoblasts, fibroblasts) can migrate (only MSCs and fibroblasts), proliferate (circular arrows), differentiate (vertical arrows), produce growth factors ( $g_{b c}$ : osteochondrogenic growth factor, $g_{v}$ : angiogenic growth factor) and extracellular matrix ( $m_{f}$ : fibrous tissue density, $m_{b}$ : bone density, $m_{c}$ : cartilage density, $m$ : total tissue density). Blood vessels are a source of oxygen ( $n$ : oxygen tension) which influences proliferation, differentiation and angiogenic growth factor production. Variables influencing a tissue level process are indicated next to the corresponding arrow. (bottom) Schematic representation of the different phases occurring during bone fracture healing. The processes that were made oxygen dependent in this study are indicated by circular arrows. 
At the cellular level, the development of the discrete vascular tree is determined by sprouting, vascular growth and anastomosis. The sprouting process is modeled in great detail by including the DII4-Notch1 signaling pathway. In short, every endothelial cell (EC) has an intracellular level of VEGFR-2, active VEGFR-2, effective active VEGFR-2, Notch1, active Notch1, effective active Notch1, DII4 and actin. The rules that capture the lateral inhibition mechanism during tip cell selection were adapted from the agent-based model of Bentley et al. (Bentley et al., 2008) and have been described in detail in Carlier et al. (Carlier et al., 2012). The growth of a blood vessel is modeled by computing the movement of the corresponding tip cell where the tip cell speed depends on the active VEGFR-2 concentration and the tip cell direction is influenced by chemotactic (angiogenic growth factor) and haptotactic (collagen fibers in the extracellular matrix) signals (Carlier et al., 2012). Note that the vessel diameter is defined by the grid resolution and is always one endothelial cell wide, whereas the movement of the tip cell is grid independent and calculated in a lattice-free manner. When a tip cell encounters another blood vessel or when it migrates outside the geometrical domain (defined in Figure 2), an anastomosis is formed during which the leading EC loses its tip cell phenotype. The newly established connection between the vessels allows for blood flow and the delivery of oxygen and nutrients. As such, only the ECs that are part of a vascular loop are sources of oxygen. This represents an important improvement with respect to the MOSAIC model where all the vessels were instantaneously active. Remark that the current oxygen model does not account for metalloproteinases that can degrade the basement membrane of the existing vessels as well as the surrounding extracellular matrix in order to invade the tissues and migrate towards the source of VEGF.

At the tissue level, the fracture healing process is described as a spatiotemporal variation of 10 continuous variables: mesenchymal stem cell density $\left(c_{m}\right)$, fibroblast density $\left(c_{f}\right)$, 
chondrocyte density $\left(c_{c}\right)$, osteoblast density $\left(c_{b}\right)$, fibrous matrix density $\left(m_{f}\right)$, cartilaginous matrix density $\left(m_{c}\right)$, bone matrix density $\left(m_{b}\right)$, generic osteochondrogenic growth factor concentration $\left(g_{b c}\right)$, vascular growth factor concentration $\left(g_{v}\right)$ and oxygen tension $(n)$. Remark that the MOSAIC model has been simplified by including only one generic osteochondrogenic growth factor $\left(g_{b c}\right)$ whose influence on differentiation is steered to either chondrogenesis or osteogenesis depending on the local oxygen tensions. The following set of partial differential equations (PDEs) of the taxis-diffusion-reaction type describes the various key processes of bone regeneration:

$$
\begin{aligned}
& \frac{\partial c_{m}}{\partial t}=\nabla[\overbrace{D_{m} \nabla c_{m}}^{\text {diffusion }}-\overbrace{C_{m_{C T} c_{m} \nabla\left(g_{b c}+g_{v}\right)}^{\text {chemotaxis }}}^{\text {chaptotaxis }}-\overbrace{C_{m_{H T} c_{m} \nabla m}}^{\text {hat }}]+\overbrace{A_{m} c_{m}\left[1-\alpha_{m} c_{m}\right]}^{\text {proliferation }} \\
& \begin{array}{lll}
\text { osteogenic } & \text { chondrogenic } & \text { fibroblastic } \\
\text { differentation } & \text { differentiation } & \text { differentiation }
\end{array} \\
& -F_{1} c_{m}-F_{2} c_{m}-\frac{F_{4} c_{m}-}{F_{7} c_{m}} \\
& \frac{\partial c_{f}}{\partial t}=\nabla[\overbrace{D_{f} \nabla c_{f}}^{\text {diffusion }}-\overbrace{C_{f} c_{f} \nabla g_{b c}}^{\text {chemotaxis }}]+\overbrace{A_{f} c_{f}\left[1-\alpha_{f} c_{f}\right]}^{\text {proliferation }}+\overbrace{F_{4} c_{m}}^{\begin{array}{c}
\text { fibroblastic } \\
\text { differentiation }
\end{array}-\overbrace{F_{3} d_{f} c_{f}}^{\begin{array}{c}
\text { endochondral } \\
\text { ossification }
\end{array}}-F_{8} c_{f} \text { apoptosis }}
\end{aligned}
$$

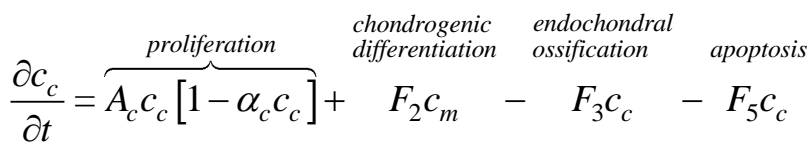

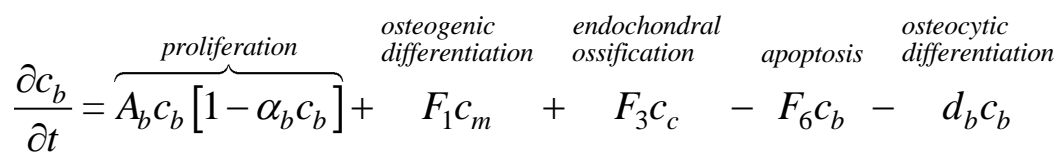

$$
\begin{aligned}
& \frac{\partial m_{f}}{\partial t}=\overbrace{P_{f s}\left(1-\kappa_{f} m_{f}\right) c_{f}}^{\text {production }}-\overbrace{Q_{f} m_{f} m_{c} c_{b}}^{\text {resorption }} \\
& \frac{\partial m_{c}}{\partial t}=\overbrace{P_{c s}\left(1-\kappa_{c} m_{c}\right) c_{c}}^{\text {production }}-\overbrace{Q_{c} m_{c} c_{b}}^{\text {resorption }} \\
& \frac{\partial m_{b}}{\partial t}=\overbrace{P_{b s}\left(1-\kappa_{b} m_{b}\right) c_{b}}^{\text {production }}
\end{aligned}
$$




$$
\begin{aligned}
& \frac{\partial g_{b c}}{\partial t}=\nabla \overbrace{\left[D_{g b c} \nabla g_{b c}\right]}^{\text {diffusion }}+\overbrace{E_{g b} c_{b}+E_{g c} c_{c}}^{\text {production }}-\overbrace{d_{g b c} g_{b c}}^{\text {denaturation }}
\end{aligned}
$$

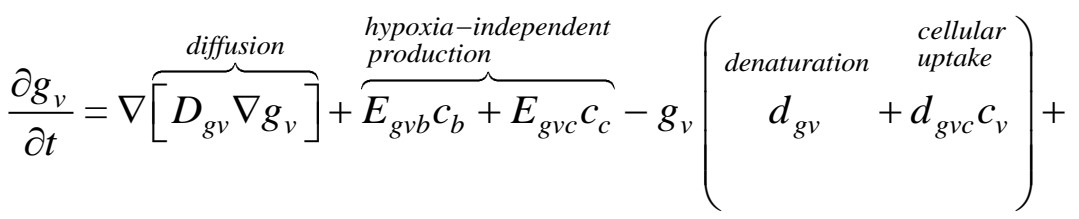

$$
\begin{aligned}
& \text { hypoxia-dependent } \\
& \text { production } \\
& \overbrace{E_{\text {hyp }, b} c_{b}+E_{\text {hyp }, \mathrm{c}} c_{c}+E_{\text {hyp }, \mathrm{f}} c_{f}+E_{\text {hyp }, \mathrm{m}} c_{m}} \\
& \frac{\partial n}{\partial t}=\nabla \overbrace{\left[D_{n} \nabla n\right]}^{\text {diffusion }}+E_{n} c_{v}-\overbrace{d_{n b} c_{b}-d_{n \mathrm{c}} c_{c}-d_{n \mathrm{f}} c_{f}-d_{n \mathrm{~m}} c_{m}}^{\text {production }}
\end{aligned}
$$

where $m\left(=m_{f}+m_{c}+m_{b}\right)$ represents the total tissue density.

After the inflammation phase, the fracture callus is filled with granulation tissue (contributing to $\left.m_{f}\right)$, MSCs $\left(c_{m}\right)$, fibroblasts $\left(c_{f}\right)$ and osteochondrogenic growth factors $\left(g_{b c}\right)$. Close to the cortex and away from the fracture gap, the MSCs will then differentiate into osteoblasts $\left(c_{b}\right)$ (intramembranous ossification $\left(m_{b}\right)$ ) whereas the central callus region will be filled with a cartilage template $\left(m_{c}\right)$ laid down by chondrocytes $\left(c_{c}\right)$. Subsequently the (hypertrophic) chondrocytes will express several angiogenic growth factors $\left(g_{v}\right)$, of which VEGF is the most important one, in order to attract blood vessels and osteoblasts. The cartilage template is gradually resorbed and replaced by woven bone (endochondral ossification $\left.\left(m_{b}\right)\right)$. Finally, the newly formed bone is remodeled, a process that is, however, not included in the model.

\subsubsection{Oxygen dependent model terms}

The essence of the new oxygen model is an accurate description of the oxygen dependency of a number of cellular processes, namely osteogenic and chondrogenic differentiation, cell proliferation, cell death, oxygen consumption and the hypoxia-dependent production of an 
angiogenic growth factor. An overview of the oxygen values at which the considered cellspecific oxygen-dependent processes occur at maximal rate or at which their rate changes, is given in Table 1. Only the functional forms that were adapted (compared to (Carlier et al., 2012)) in order to account for the influence of oxygen are discussed below, also providing adequate reference to the experimental work that forms the basis for the parameter values that appear in these forms. A description of the unaltered functional forms related to migration $\left(D_{m}, C_{m C T}, C_{m H T}, C_{f}\right)$ as well as the complete set of equations, nondimensionalized parameters, boundary and initial conditions, parameter values and implementation details can be found in Carlier et al. (Carlier et al., 2012), Peiffer et al. (Peiffer et al., 2011) and Geris et al. (Geris et al., 2008) and are provided here as supplementary material.

Table 1: Overview of the oxygen tensions at which the rate of distinct cellular processes is maximal or changes. The average initial oxygen tension is put in bold. Biological processes that preferentially take place in low oxygen tensions (upper part of Table 1) will occur in regions where the oxygen tensions will have dropped with respect to the initial value (e.g. the central fracture zone) while biological processes that preferentially take place in high oxygen tensions (lower part of Table 1 ) will occur in regions where the oxygen tensions will have increased with respect to the initial value (e.g. near the blood vessels of the periosteal layer).

\begin{tabular}{|c|c|c|}
\hline Process & $\begin{array}{l}\text { Oxygen } \\
\text { tension }\end{array}$ & Reference \\
\hline $\begin{array}{l}\text {-chondrocyte cell death }\left(F_{5,1}\right) \\
\text {-MSC cell death }\left(F_{7}\right)\end{array}$ & $0.5 \%$ & $\begin{array}{l}\text { (Henrotin et al., 2005;Grimshaw } \\
\text { and Mason, 2000) }\end{array}$ \\
\hline $\begin{array}{l}\text {-hypoxia-dependent angiogenic growth factor } \\
\text { production by chondrocytes }\left(E_{h y p, c}\right) \\
\text {-oxygen tension at half maximal } \\
\text { oxygen consumption rate of chondrocytes }\left(d_{n c}\right)\end{array}$ & $1.5 \%$ & estimated \\
\hline $\begin{array}{l}\text { - hypoxia-dependent angiogenic growth factor } \\
\text { production by MSCs }\left(E_{h y p, m}\right) \\
\text {-oxygen tension at half maximal } \\
\text { oxygen consumption rate of MSCs }\left(d_{n m}\right) \\
\text {-osteoblast cell death }\left(F_{6}\right)\end{array}$ & $2 \%$ & $\begin{array}{l}\text { (Fraisl et al., 2009;Chae et al., } \\
\text { 2001) }\end{array}$ \\
\hline -fibroblast cell death $\left(F_{8}\right)$ & $2.25 \%$ & estimated \\
\hline $\begin{array}{l}\text { - chondrogenic differentiation }\left(F_{2}\right) \\
\text { - chondrocyte proliferation }\left(A_{c}\right)\end{array}$ & $3 \%$ & $\begin{array}{l}\text { (Hirao et al., 2006; Kanichai et } \\
\text { al., 2008;Merceron et al., 2010) }\end{array}$ \\
\hline $\begin{array}{l}\text { - MSC proliferation }\left(A_{m}\right) \\
\text { - average initial oxygen tension } \\
\text { - hypoxia-dependent angiogenic growth factor } \\
\text { production by osteoblasts }\left(E_{h y p, b}\right)\end{array}$ & $4 \%$ & $\begin{array}{l}\text { (Xu et al., 2007;Grayson et al., } \\
\text { 2007; Lennon et al., 2001;Ren et } \\
\text { al., 2006;Brighton and Krebs, } \\
\text { 1972) }\end{array}$ \\
\hline
\end{tabular}




\begin{tabular}{|c|c|c|}
\hline $\begin{array}{l}\text {-oxygen tension at half maximal } \\
\text { oxygen consumption rate of osteoblasts }\left(d_{n b}\right)\end{array}$ & & estimated \\
\hline $\begin{array}{l}\text { - hypoxia-dependent angiogenic growth factor } \\
\text { production by fibroblasts }\left(E_{h y p, f}\right) \\
\text {-oxygen tension at half maximal } \\
\text { oxygen consumption rate of fibroblasts }\left(d_{n f}\right)\end{array}$ & $4.5 \%$ & estimated \\
\hline $\begin{array}{l}\text { - osteoblast proliferation }\left(A_{b}\right) \\
\text { - osteogenic differentiation }\left(F_{1}\right) \\
\text { - endochondral ossification }\left(F_{3}\right)\end{array}$ & $8 \%$ & (Hirao et al., 2006) \\
\hline - fibroblast proliferation $\left(A_{f}\right)$ & $9 \%$ & estimated \\
\hline -chondrocyte cell death $\left(F_{5,2}\right)$ & $11 \%$ & (Cheema et al., 2008) \\
\hline -maximal oxygen tension & $12 \%$ & (Brighton and Krebs, 1972) \\
\hline
\end{tabular}

\section{Cell differentiation}

The differentiation of MSCs towards osteoblasts is mediated by osteochondrogenic growth factors $g_{b c}$ (Gerstenfeld et al., 2003a;Cho et al., 2002) and oxygen $n$ (Hirao et al., 2006) which is mathematically modeled by the following functional form:

$$
F_{1}=\frac{Y_{11} \cdot g_{b c}}{H_{11}+g_{b c}} \cdot \frac{Y_{12} \cdot n^{6}}{I_{v}^{6}+n^{6}}
$$

A sixth-order Hill function is used to model a threshold (Bailon-Plaza and van der Meulen, 2001;Geris et al., 2008), in this case indicating that intramembranous ossification can only take place when the tension of oxygen is sufficiently high (i.e. $I_{v}$ is equal $8 \%$ ) (Hirao et al., 2006). Remark that the influence of the angiogenic growth factor $\left(g_{v}\right)$ (Street et al., 2002) on osteogenic differentiation has been omitted in this study since it has become obsolete due to the addition of the discrete blood vessels and oxygen.

The differentiation of MSCs to chondrocytes is modeled as follows:

$$
F_{2}=\frac{Y_{2} g_{b c}}{H_{2}+g_{b c}} \cdot \frac{Y_{2 n} n}{K_{2 n}^{6}+n^{6}}
$$

where the optimal oxygen tension is $3 \%$. This value was chosen in accordance to experimental values found in literature. In low oxygen environments ( $2 \%$ oxygen tension) 
Malladi et al. observed a decrease in chondrogenesis (Malladi et al., 2006) whereas a more recent study by Kanichai et al. reports that a low oxygen environment ( $2 \%$ oxygen tension) has a beneficial effect on chondrogenesis, measured by a significant increase in collagen II expression and proteoglycan deposition with respect to normoxic (20\% oxygen) conditions (Kanichai et al., 2008). Hirao et al. showed that $5 \%$ oxygen tension promoted chondrogenic commitment rather than osteoblastic differentiation (Hirao et al., 2006). Also the data of Merceron et al. strongly suggest that hypoxia (5\% oxygen tension) favors the chondrogenic differentiation of human adipose tissue-derived stem cells (Merceron et al., 2010). Hence, an intermediate value for the optimal oxygen tension for chondrogenic differentiation was chosen.

\section{Endochondral ossification}

The functional form of the endochondral ossification term $\left(F_{3}\right)$ is taken from Carlier et al. (Carlier et al., 2012). Remark that its parameter values have changed (i.e. oxygen switch $B_{v}$ is set at $8 \%$ ) so that they correspond to the optimal oxygen tension for osteoblast proliferation (see below) and (osteogenic) differentiation.

$$
F_{3}=\frac{m_{c}^{6}}{B_{e c}^{6}+m_{c}^{6}} \cdot \frac{Y_{3} g_{b c}}{H_{3}+g_{b c}} \cdot \frac{n^{6}}{B_{v}^{6}+n^{6}}
$$

\section{Cell proliferation}

In this work the proliferation of all cell types (i.e. $c_{m}, c_{f}, c_{b}$ and $c_{c}$ ) is modeled similar to Carlier et al. (Carlier et al., 2012) except that the influence of oxygen is taken into account explicitly:

$$
A_{i}=\frac{A_{i 0} m}{K_{i}^{2}+m^{2}} \cdot \frac{A_{i n} n}{K_{i n}^{3}+n^{3}}
$$


with $i=m$ for $\operatorname{MSCs}\left(c_{m}\right)$ and

$$
A_{i}=\frac{A_{i 0} m}{K_{i}^{2}+m^{2}} \cdot \frac{A_{i n} n}{K_{i n}^{2}+n^{2}}
$$

with $i=f$ for fibroblasts $\left(c_{f}\right)$ and

$$
A_{i}=\frac{A_{i 0} m}{K_{i}^{2}+m^{2}} \cdot \frac{A_{i n} n}{K_{i n}^{6}+n^{6}}
$$

with $i=c, b$ for chondrocytes $\left(c_{c}\right)$ and osteoblasts $\left(c_{b}\right)$. The optimal oxygen tension for MSC proliferation $\left(A_{m}\right)$ is set at $4 \%$, based on experimental values in the literature. Grayson et al. report a 9-fold increase in the amount of human bone marrow derived MSCs obtained after culture at $2 \%$ oxygen with respect to a 5 -fold increase at $20 \%$ oxygen (Grayson et al., 2007). These results are similar to the findings of Ren et al. who report a 2.8 -fold increase in the proliferation of murine bone marrow cells under $8 \%$ oxygen in comparison to their expansion under $21 \%$ oxygen (Ren et al., 2006). Also, Lennon et al. report $50 \%$ more cell yield of rat bone marrow derived MSCs in $5 \%$ oxygen compared to $20 \%$ oxygen (Lennon et al., 2001). Moreover, murine adipose derived MSCs are reported to yield a significantly higher cell number due to a reduction in doubling time at $2 \%$ oxygen (Xu et al., 2007). The optimal oxygen tension for fibroblast proliferation $\left(A_{f}\right)$ is set at $9 \%$ since Fermor et al. found that human anterior cruciate ligament-derived fibroblasts proliferated maximally at $10 \%$ oxygen, moderately at $21 \%$ and much slower at $0 \%$ (Fermor et al., 1998). The third and second order denominator in $A_{m}$ and $A_{f}$ was used to describe a broad distribution, indicating that the proliferative capacity of MSCs and fibroblasts is only moderately oxygen dependent (compared to the sixth order denominator for osteoblasts and chondrocytes, see below). The optimal oxygen tension for chondrocyte proliferation $\left(A_{c}\right)$ is set at $3 \%$, corresponding to the optimal oxygen tension for chondrocyte differentiation $\left(F_{2}\right)$. Similarly, the optimal 
oxygen tension for osteoblast proliferation $\left(A_{b}\right)$ is set at $8 \%$, corresponding to the optimal oxygen tension for osteoblast differentiation and endochondral ossification $\left(F_{1}\right.$ and $\left.F_{3}\right)$. A switch-like behavior was introduced in the functional forms of $A_{b}$ and $A_{c}$ by using a sixthorder denominator (Bailon-Plaza and van der Meulen, 2001;Geris et al., 2008). Remark that the chosen oxygen parameter values for cellular proliferation are all within the experimental range of oxygen tensions measured in rabbit fibular fractures: $0.8 \%$ in the hematoma, $9 \%$ in fibrous tissue, $4.5 \%$ in cartilage, $4-12 \%$ in fibrous bone and $12.2-14 \%$ in diaphysal bone (Brighton et al., 1991).

\section{Oxygen consumption}

In order to perform the multiple basic cellular processes required e.g. for fracture healing, cells need oxygen (Lu et al., 2013a). In this study we have replaced the general oxygen decay term of the MOSAIC model with a cell-specific description of cellular oxygen consumption. Similar to Demol et al., the cellular consumption of oxygen was described using a MichaelisMenten kinetic law (Demol et al., 2011):

$$
d_{n i}=\frac{Q_{n i} n}{K_{n i}+n}
$$

with $i=m, f, b, c$ for MSCs, fibroblasts, osteoblasts and chondrocytes respectively. The oxygen tension at half maximal-consumption rate $\left(K_{n i}\right)$ was chosen half-way between the oxygen value for optimal proliferation and cell death (see below). Moreover, this oxygen tension was taken equal to the lower oxygen limit at which hypoxia signaling is activated, resulting in hypoxia-dependent production of angiogenic growth factor $\left(g_{v}\right)$ (see also below). In order to determine the parameter values of the maximal oxygen consumption rate $\left(Q_{n i}\right)$, the non-dimensionalized parameter values were fitted so that the correspondence with the 
experimentally observed aspects of fracture healing, i.e. the spatial patterns of cells and tissues, was improved thereby keeping in mind the following relative order of oxygen consumption: $Q_{n c}<Q_{n m}<Q_{n b}<Q_{n f}$ (Table 2).

Table 2: Comparison between the consumption rates used in the oxygen model and the experimentally measured values.

\begin{tabular}{|c|c|c|}
\cline { 2 - 3 } \multicolumn{1}{c|}{} & $\begin{array}{c}\text { Consumption rate used in } \\
\text { oxygen model (mol/cell.s) }\end{array}$ & $\begin{array}{c}\text { Experimentally measured } \\
\text { consumption rate (mol/cell.s) }\end{array}$ \\
\hline chondrocytes $\left(Q_{n c}\right)$ & $0.5 \times 10^{-18}$ & $0.2-4 \times 10^{-18}$ (Malda et al., 2004a) \\
\hline $\operatorname{MSCs}\left(Q_{n m}\right)$ & $23.2 \times 10^{-18}$ & $38 \times 10^{-18}$ (Cochran et al., 2006) \\
\hline osteoblasts $\left(Q_{n b}\right)$ & $25.5 \times 10^{-18}$ & $11.1 \times 10^{-18}$ (Komarova et al., 2000) \\
\hline fibroblasts $\left(Q_{n f}\right)$ & $29 \times 10^{-18}$ & $370 \times 10^{-18}$ (Papandreou et al., 2006) \\
\hline
\end{tabular}

Remark that the endothelial cells are not included in Table 2 since they mostly rely on anaerobic metabolism for energy production thus consuming very little oxygen (Peters et al., 2009;Mertens et al., 1990;De Bock et al., 2013).

\section{Hypoxia-dependent production of angiogenic growth factor}

In low oxygen environments, the transcription factor HIF-1 $\alpha$ is stabilized and translocates to the nucleus where it forms a complex with the HIF-1 13 subunit in order to promote the transcription of genes with a hypoxia responsive element (HRE), such as the angiogenic growth factor VEGF (Pugh and Ratcliffe, 2003;Maes et al., 2012). In the oxygen model we make a distinction between hypoxia-independent production of the angiogenic growth factor $\left(g_{v}\right)$ (by hypertrophic chondrocytes and osteoblasts) and hypoxia-dependent production (by MSCs, osteoblasts, fibroblasts and chondrocytes), the latter being modeled as follows:

$$
E_{\text {hyp }, i}=\frac{Q_{\text {hyp }, i} K^{6}{ }_{h y p, i}}{K_{h y p, i}^{6}+n^{6}}
$$


with $i=m, f, b, c$ for MSCs, fibroblasts, osteoblasts and chondrocytes respectively. A threshold-like behavior was introduced in the functional form of $E_{h y p, i}$ by using a sixth-order formulation (Bailon-Plaza and van der Meulen, 2001;Geris et al., 2008). Due to the lack of quantitative data on the hypoxia-dependent angiogenic growth factor production rate $\left(Q_{h y p}\right)$ of distinct skeletal cell types, the parameter value was chosen equal for all cell types and set at $0.1 \mathrm{pg} /$ day.cell. Although this parameter value is higher than reported in literature (16-33 x $10^{-3} \mathrm{pg} /$ day.cell for fibroblasts (Zelzer et al., 2001) and $750 \times 10^{-6} \mathrm{pg} /$ day.cell for muscle cells (Kubo et al., 2009)), it represents the maximal production rate which is modulated by the local oxygen tension, thereby increasing the correspondence with the experimentally reported values. The oxygen threshold at which the cells switch on their HIF-signaling pathway $\left(K_{\text {hyp }}\right)$ was chosen equal to the oxygen tension at half maximal-consumption rate $\left(K_{n i}\right)$.

\section{Cell death}

On the one hand, in pathologically low oxygen environments, cellular metabolism is compromised resulting in cell death (Cheema et al., 2008). A new functional form describing the oxygen-dependent cell death of chondrocytes $\left(F_{5}\right)$, osteoblasts $\left(F_{6}\right), \operatorname{MSCs}\left(F_{7}\right)$ and fibroblasts $\left(F_{8}\right)$ is introduced:

$$
F_{5}=F_{5,1}+F_{5,2}=\frac{A_{5 n 1} H_{5 n 1}{ }^{6}}{H_{5 n 1}{ }^{6}+n^{6}}+\frac{A_{5 n 2} n^{6}}{H_{5 n 2}{ }^{6}+n^{6}}
$$

for chondrocytes and

$$
F_{6,7,8}=\frac{A_{i n} H_{i n}{ }^{6}}{H_{i n}{ }^{6}+n^{6}}
$$


with $\mathrm{i}=6,7,8$ for osteoblasts, MSCs and fibroblasts respectively. Although chondrocytes are well adapted to low oxygen tensions, they do require a minimal oxygen level for their basal metabolic functions (Henrotin et al., 2005). Indeed, Grimshaw et al. show that oxygen tensions below $1 \%$ inhibited glucose uptake and lactate production as well as cellular ribonucleic acid synthesis in bovine articular chondrocytes (Grimshaw and Mason, 2000). Consequently, a sharp switch at $0.5 \%$ oxygen tension was used to model chondrocyte death. The same threshold was used for mesenchymal stem cells, since experiments have evidenced their ability to survive long periods of nutrient and oxygen deprivation (Deschepper et al., 2011;Potier et al., 2007). Osteoblasts, in contrast to mesenchymal stem cells, are reported to produce less collagen and mineralized matrix in hypoxic conditions ( $2 \%$ oxygen tension)(Nicolaije et al., 2012). Moreover, an increase in osteoblast death is seen starting from 2\% oxygen tension (Nicolaije et al., 2012;Chae et al., 2001), justifying the model threshold of $2 \%$ oxygen tension for osteoblast death. As no data exist on the oxygen tension at which fibroblasts die, this was chosen comparable to that of osteoblasts $(2.25 \%$ oxygen tension).

In pathologically high oxygen environments, on the other hand, very high oxygen tensions induce oxidative stress due to mitochondrial respiration (Cheema et al., 2008), whereby chondrocytes are less protected against reactive oxygen species (ROS). This was shown by Brandl et al. who investigated the (stress) response of human osteoarthritic chondrocytes subjected to acute or prolonged oxidant challenge with hydrogen peroxide (Brandl et al., 2011). Consequently, the death term of chondrocytes consists of two contributions: increased cell death both in very low $(0.5 \%$ oxygen tension) and high oxygen tensions ( $11 \%$ oxygen tension) (Henrotin et al., 2005). Remark that the other cell types only have one term, 
i.e. they can survive in well oxygenated environments (bearing in mind that in the model simulations oxygen tension is not exceeding $12 \%)$.

\subsubsection{Growth factor dependent model terms}

In the oxygen model the chondrogenic growth factor $\left(g_{c}\right)$ and osteogenic growth factor $\left(g_{b}\right)$ that influenced chondrogenic and osteogenic differentiation respectively in the MOSAIC model are merged into one generic osteochondrogenic growth factor $\left(g_{b c}\right)$ whose influence on differentiation is steered to either chondrogenesis or osteogenesis by oxygen. This simplification was inspired by the recent experimental findings that several growth factors (i.e. BMP-2 and BMP-6) have both osteogenic as well as chondrogenic properties (Kwon et al., 2013;Hojo et al., 2013). The chondrogenic and osteogenic growth factors $g_{c}$ and $g_{b}$ were replaced by $g_{b c}$ in all the functional forms containing them (i.e. $F_{1}, F_{2}, F_{3}, E_{g b}, E_{g c}, C_{m c t}, C_{f}$ ), maintaining the mathematical formulation but changing some of the parameter values in order to match the predicted and reported dynamics of bone regeneration (Equations 11-13, 21-24 and Table S1 in the supplementary material). The boundary conditions are also kept the same and an initial condition $\left(g_{b c, i n i t}=100 \mathrm{ng} / \mathrm{ml}\right)$ was introduced.

$$
\begin{aligned}
& E_{g b}=\frac{G_{g b} g_{b c}}{H_{g b}+g_{b c}} \\
& E_{g c}=\frac{G_{g c} g_{b c}}{H_{g c}+g_{b c}} \cdot \frac{m}{K_{g c}^{3}+m^{3}} \\
& C_{m_{C T}}=\frac{C_{k C T m}\left(g_{b c}+g_{v}\right)}{K_{k C T m}^{2}+\left(g_{b c}+g_{v}\right)^{2}} \\
& C_{f}=\frac{C_{k f} g_{b c}}{K_{k f}^{2}+g_{b c}^{2}}
\end{aligned}
$$




\subsection{Implementation details}

The set of partial differential equations (Equations 1-10) is numerically solved by the method of lines (MOL). Firstly, a finite volume method is used to spatially discretize the PDEs, assuring mass conservation and non-negativity of the continuous variables (Gerisch and Chaplain, 2006). Secondly, the resulting set of ODEs is integrated in time using ROWMAP, a ROW-code of order four with Krylov techniques for large stiff ODEs (Weiner et al., 1997). After each time step for the continuous variables, the positions of the discrete ECs as well as their intracellular levels are updated (see (Peiffer et al., 2011) and (Carlier et al., 2012) for details). The PDE model is solved on a 2D grid with a grid cell size of $25 \mu \mathrm{m}$, equal to the width of a discrete endothelial cell. The fiber orientation, which accounts for the haptotactic guidance of tip cell movement, is randomly initialized. For the same initial settings of fiber orientation, the model is deterministic. However, some stochasticity in the movement of the ECs can be easily introduced by altering the random orientation of the fibers (Qutub and Popel, 2009). The oxygen model is implemented in Matlab (The MathWorks, Natick, MA).

\subsection{Simulation details}

The simulations were performed on a quad-core Intel ${ }^{\circledR}$ Xeon ${ }^{\circledast} \mathrm{CPU}$ with 12 GB RAM memory.

\subsubsection{Normal fracture healing}

The geometrical domain is deduced from the real callus geometry at three weeks post fracture in a standardized femoral rat fracture model (Harrison et al., 2003). Due to reasons of symmetry, only one-fourth of the domain is simulated. Initially the callus domain is filled with granulation tissue, which is, since it is not explicitly modeled as a separate phase, represented here by a small initial amount of fibrous tissue $\left(m_{f, \text { init }}=10 \mathrm{mg} / \mathrm{ml}\right)$, osteochondrogenic growth factors $\left(g_{b c, \text { init }}=100 \mathrm{ng} / \mathrm{ml}\right)$, mesenchymal stem cells $\left(c_{m, \text { init }}=\right.$ 
$2.10^{4}$ cells $/ \mathrm{ml}$ ) and fibroblasts $\left(c_{f, \text { init }}=1.10^{4}\right.$ cells $\left./ \mathrm{ml}\right)$. Due to the rupturing of the blood vessels during the fracture, the oxygen tension in the fracture callus will gradually decrease. We consider an initial gradient of oxygen tension $n_{\text {init }}=3.7[\%]+2.33\left[\frac{\%}{\mathrm{~mm}}\right] . \mathrm{X}[\mathrm{mm}]$ with X representing the coordinate on the horizontal axis in Figure 2. This gradient was chosen so that the oxygen tension is lowest in the fracture gap (3.7\% oxygen tension) and highest near the bone ends (5.8\% oxygen tension) since the latter region is close to blood vessels supplying it with oxygen. The average initial oxygen tension is $4 \%$, similar to what is measured for cartilage and bone marrow (Brighton and Krebs, 1972;Epari et al., 2008;Fraisl et al., 2009). The influence of this initial gradient was investigated in a sensitivity analysis (see further). The boundary conditions are chosen similar to the MOSAIC model (Carlier et al., 2012), except that both the chondrogenic $\left(g_{c}\right)$ and osteogenic $\left(g_{b}\right)$ growth factors are replaced by one general osteochondrogenic growth factor $\left(g_{b c}\right)$. The geometry of the fracture callus, as well as the boundary conditions and initial positions of the endothelial cells are represented in Figure 2.
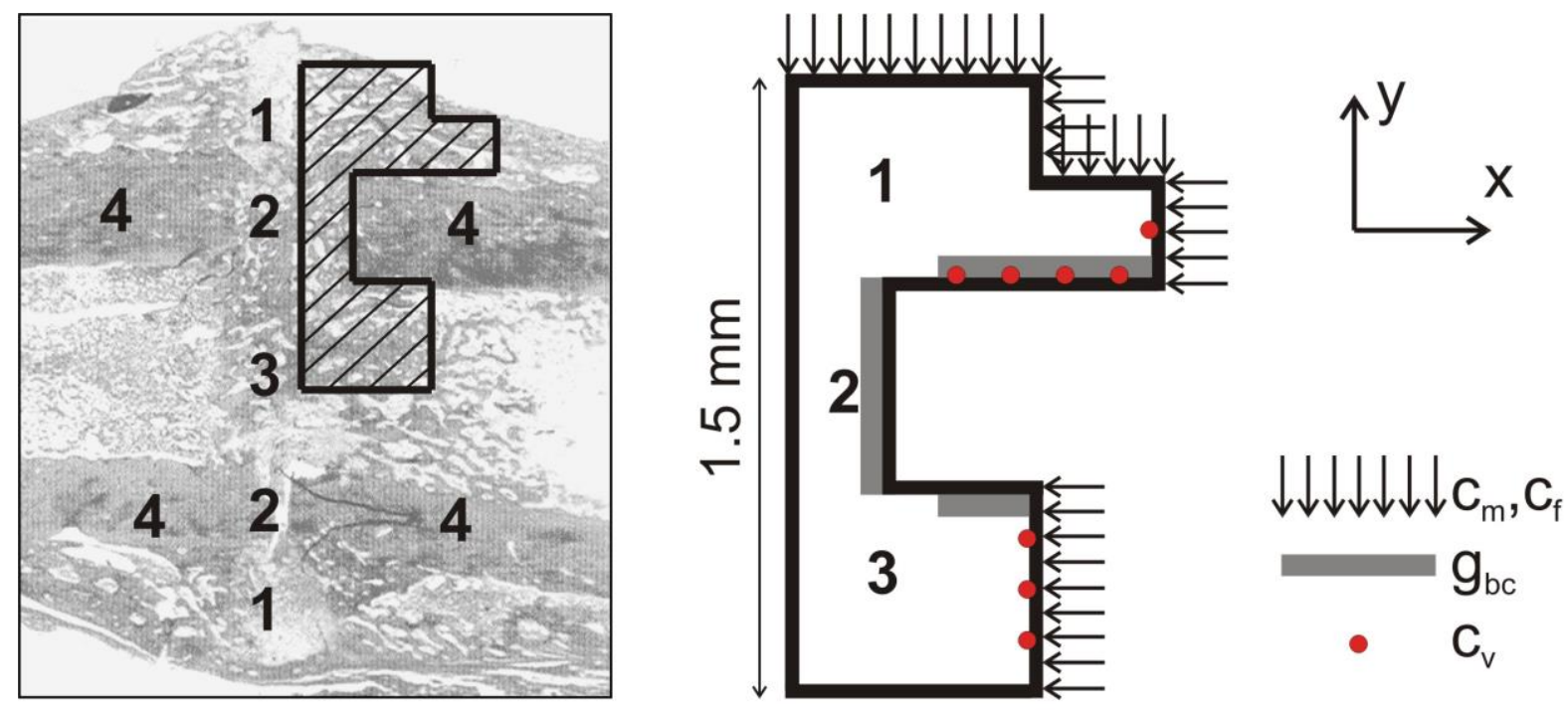

Figure 2: (left) The geometrical domain models one-fourth of the real fracture callus geometry (Harrison et al., 2003) due to reasons of symmetry; 1 periosteal callus; 2 
intercortical callus; 3 endosteal callus; 4 cortical bone ends. (right) No-flux boundary conditions are assumed for all variables, except for the mesenchymal stem cells $\left(c_{m}\right)$ and fibroblasts $\left(c_{f}\right)$ which are released from the periosteum, surrounding soft tissues and bone marrow (Gerstenfeld et al., 2003b); and the osteochondrogenic growth factor $\left(g_{b c}\right)$ which is released from the degrading bone ends and the cortex (Barnes et al., 1999;Dimitriou et al., 2005). The origin of the coordinate system is placed in the left bottom corner of the geometrical domain.

\subsubsection{Sensitivity analysis}

Peiffer et al. (Peiffer et al., 2011) previously performed an extensive convergence analysis on the time step size and grid cell size as well as a sensitivity analysis on the orientation of the matrix fibers, the initial tip cell density and ratio of chemotaxis to haptotaxis influencing tip cell speed. Consequently, in this study the additional sensitivity analyses focused on the influence on the simulation outcome of the initial conditions $\left(\tilde{m}_{f, \text { init }}, \tilde{g}_{b c \text {,init }}, \tilde{c}_{m, \text { init }}, \tilde{c}_{f, \text { init }}\right.$, $\left.\tilde{n}_{\text {init }}\right)$, boundary conditions $\left(\tilde{g}_{b c, B C}, \tilde{c}_{m, B C}, \tilde{c}_{f, B C}\right)$ and the oxygen thresholds in the newly defined functional forms $\left(A_{m}, A_{f}, A_{c}, A_{b}, F_{1}, F_{2}, F_{3}, d_{n m}, d_{n c}, d_{n b}, d_{n f}, E_{h y p, m}, E_{h y p, c}, E_{h y p, b}, E_{h y p, f}, F_{5}\right.$, $\left.F_{6}, F_{7}, F_{8}\right)$. The values of the initial conditions and boundary conditions were varied between $50 \%$ and $150 \%$ of the original parameter values. In order to test the sensitivity of the model outcome to the oxygen parameter values listed in Table 1, these values were varied with $1 \%$ oxygen tension, at the same time maintaining the correct order of oxygen tension values at which distinct cellular processes occur within one cell type (e.g. the oxygen tension for proliferation should not be below the oxygen tension for cell death for a specific cell type) and maintaining the overall value for the respective functional form so that only the effect of the threshold is tested. 


\subsubsection{Impaired fracture healing}

The gap size of the newly developed oxygen model was enlarged from $0.5 \mathrm{~mm}$ to $3 \mathrm{~mm}$ in order to simulate and investigate critically sized defects. This gap size was chosen in accordance with the experimental observations of Harrison et al. who report the formation of a pseudarthrosis in a $3 \mathrm{~mm}$ distracted mid-diaphysal rat femoral osteotomy (Harrison et al., 2003). This value is also in the same range as other rat femoral critical defect sizes reported in literature: $1 \mathrm{~cm}$ (Vogelin et al., 2005), $8 \mathrm{~mm}$ (Tolli et al., 2011), $6 \mathrm{~mm}$ (Drosse et al., 2008). 


\section{Results}

\subsection{Normal fracture healing}

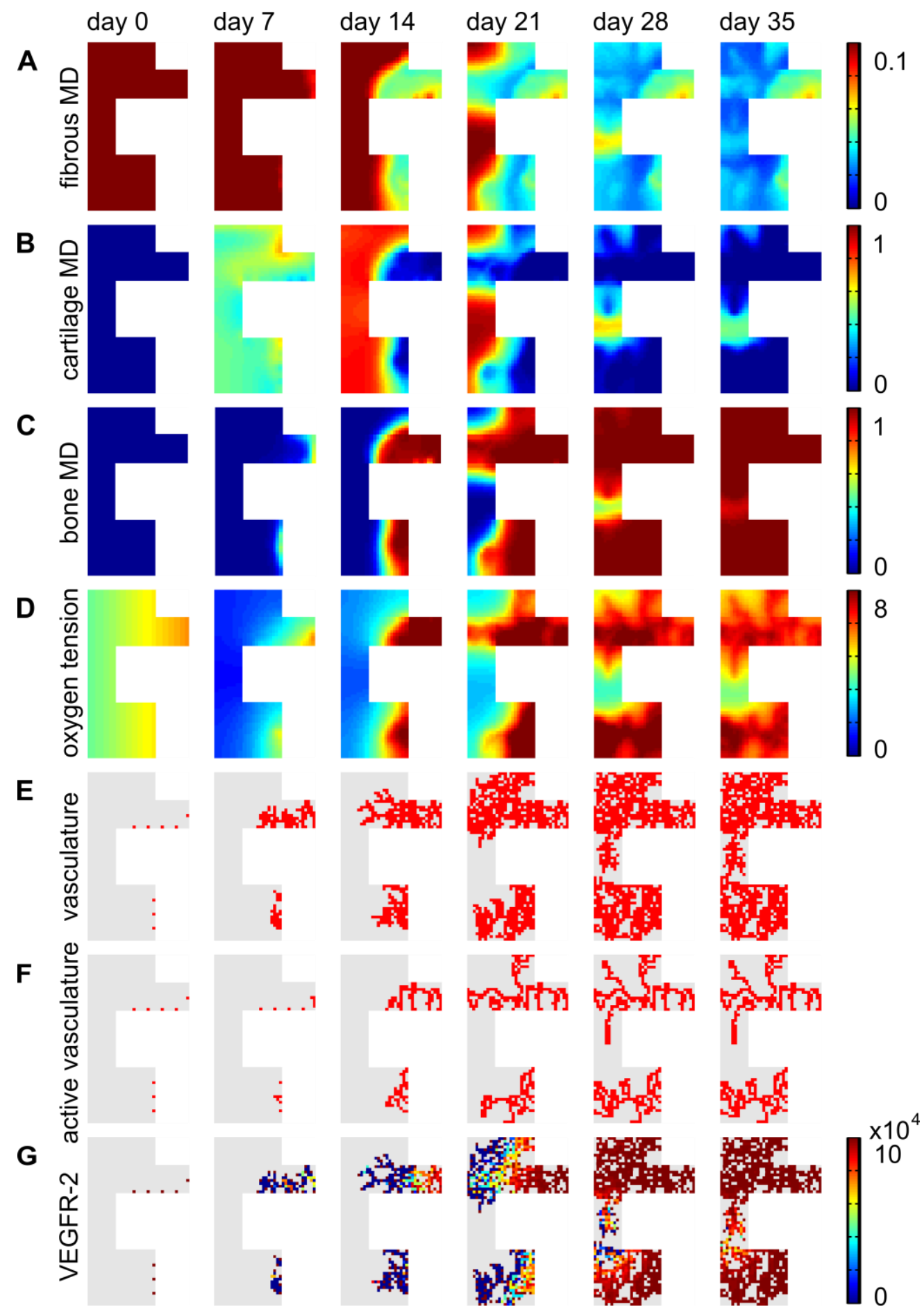

Figure 3: The spatiotemporal evolution of (A) fibrous tissue matrix density (MD) (x 0.1 $\mathrm{g} / \mathrm{ml})$, (B) cartilage matrix density $(x 0.1 \mathrm{~g} / \mathrm{ml}),(C)$ bone matrix density $(x 0.1 \mathrm{~g} / \mathrm{ml}),(D)$ oxygen tension ( $x$ 1\%), $(E)$ vasculature, $(F)$ active vasculature and $(G)$ VEGFR-2 levels on the EC during normal fracture healing in a small defect $(0.5 \mathrm{~mm})$ as predicted by the oxygen model. 
The oxygen model captures the essential features of the fracture healing process (Figure 3) starting with osteoprogenitor cells $\left(c_{m}\right)$ entering the callus from the periosteal layer and differentiating into osteoblasts $\left(c_{b}\right)$ near the cortex and chondrocytes $\left(c_{c}\right)$ in the intercortical callus. This leads to intramembranous ossification near the bony ends and endochondral ossification in the rest of the fracture zone. The evolution of the fracture healing process as predicted by the oxygen model is very similar to the evolution predicted by the previously published MOSAIC model (figure S.2), except for the fibrous tissue density and the cartilage density. For the first, a quicker resorption is seen in the oxygen model and specifically in the endosteal callus (Figure $3 \mathrm{~A}$ ). For the latter, a more homogeneous distribution is predicted by the oxygen model (Figure 3B). In the current oxygen model only the ECs that are part of a vascular loop are sources of oxygen. This implementation results in an active vasculature, i.e. the functional vessels that deliver oxygen, that is characterized by a density that is much lower than the overall vascular density (Figure 3E-F), resembling more the final, remodeled vascular tree. 


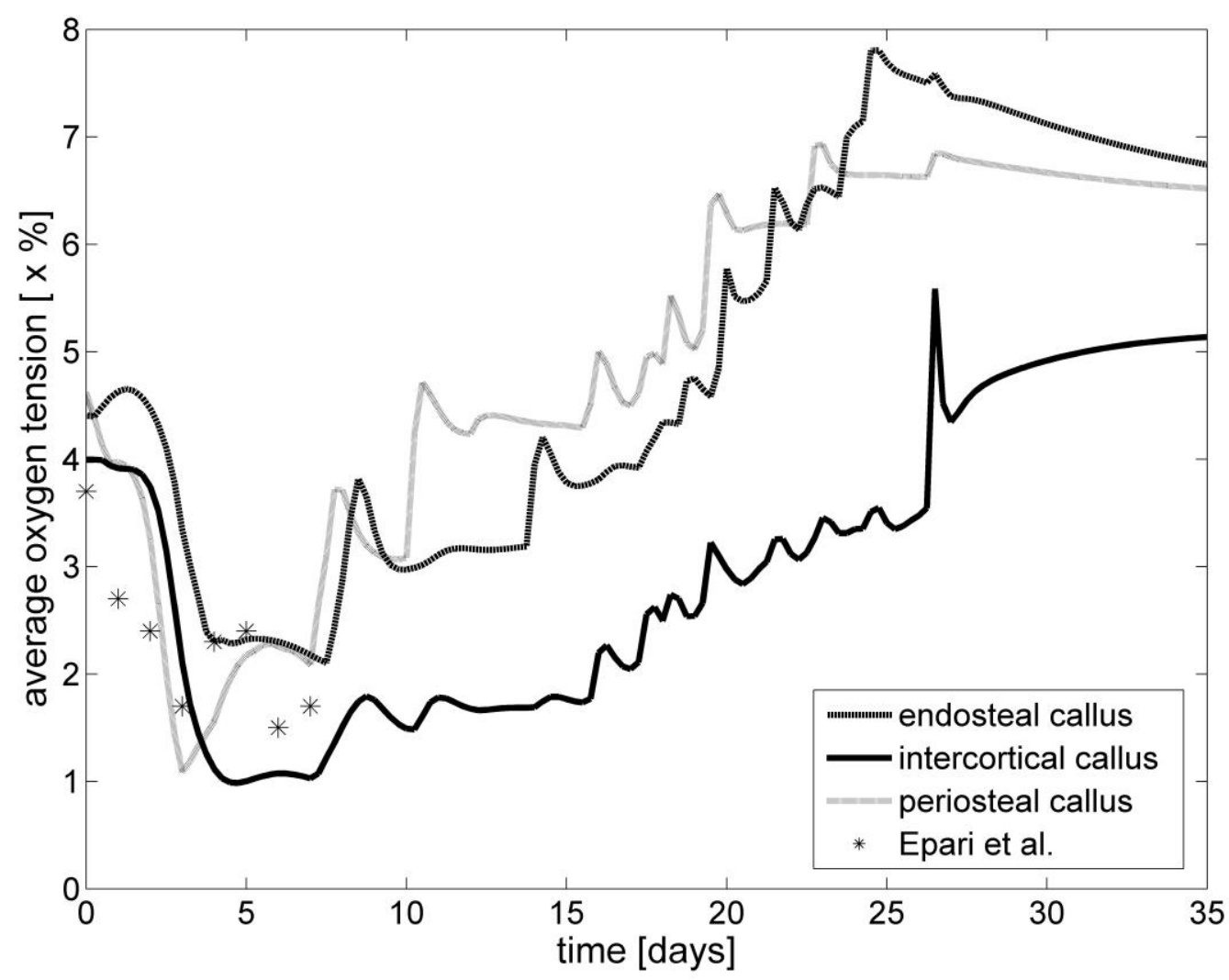

Figure 4: Temporal evolution of the average oxygen tension in the endosteal (--), intercortical (-) and periosteal (...) callus as predicted by the oxygen model. * indicates the experimentally measured oxygen tensions in an ovine tibial osteotomy (Epari et al., 2008). Since Epari et al. start to measure at post fracture day (PFD) 0 and the oxygen model neglects the inflammation phase, the experimental measurements were shifted with three days, i.e. the experimental point measured at day 3 is depicted here at day 0 .

The complex interplay between oxygen delivery, oxygen diffusion and oxygen consumption during the bone regeneration process gives rise to interesting oxygen patterns (Figure 4). The initial average oxygen tension of around $4 \%$ quickly drops due to cellular consumption, comparable to the experimental temporal dynamics observed by Epari et al. (indicated by * in Figure 4) (Epari et al., 2008). Epari et al. measured the oxygen tensions in an ovine tibial osteotomy model continuously for 10 days post fracture, reporting an initial oxygen tension of $110 \mathrm{mmHg}(\sim 14.6 \%)$ and a final oxygen tension of $12.5 \mathrm{mmHg}(\sim 1.65 \%)$. Since the oxygen model does not include the inflammation phase, the initial oxygen tension in the oxygen model represents the oxygen tension after three days. Epari et al. measured an oxygen 
tension of $28 \mathrm{mmHg}$ after 3 days ( $3.7 \%$ ) which is in accordance with the value used in the oxygen model ( $4 \%$, Table 1$)$. Remark that the first three data points (PFD 0, 1, 2) of Epari et al. are not indicated in order to facilitate the interpretation of Figure 4.

As the newly formed vessels grow into the fracture site, the oxygen tension gradually increases from PFD 7 on. After 35 days the average oxygen tension is between $5 \%$ and $7 \%$. Remark that these are average values over the respective calluses. Indeed, the maximal oxygen tensions attained in the periosteal, endosteal and intercortical callus are $11 \%, 9 \%$ and $7 \%$ respectively. The average oxygen profiles in Figure 4 are not smooth and show a lot of temporal variation. This is due to the discontinuous process of vascular loop formation. In some locations an anastomosis will be formed, resulting in the local delivery of oxygen by that vascular loop. This might, however, boost the local proliferation of cells, resulting in a lowering of the oxygen tension due to the increased oxygen consumption. Remark that the lowest oxygen values are found in the intercortical callus, since this part is furthest away from the ingrowing blood vessels. 

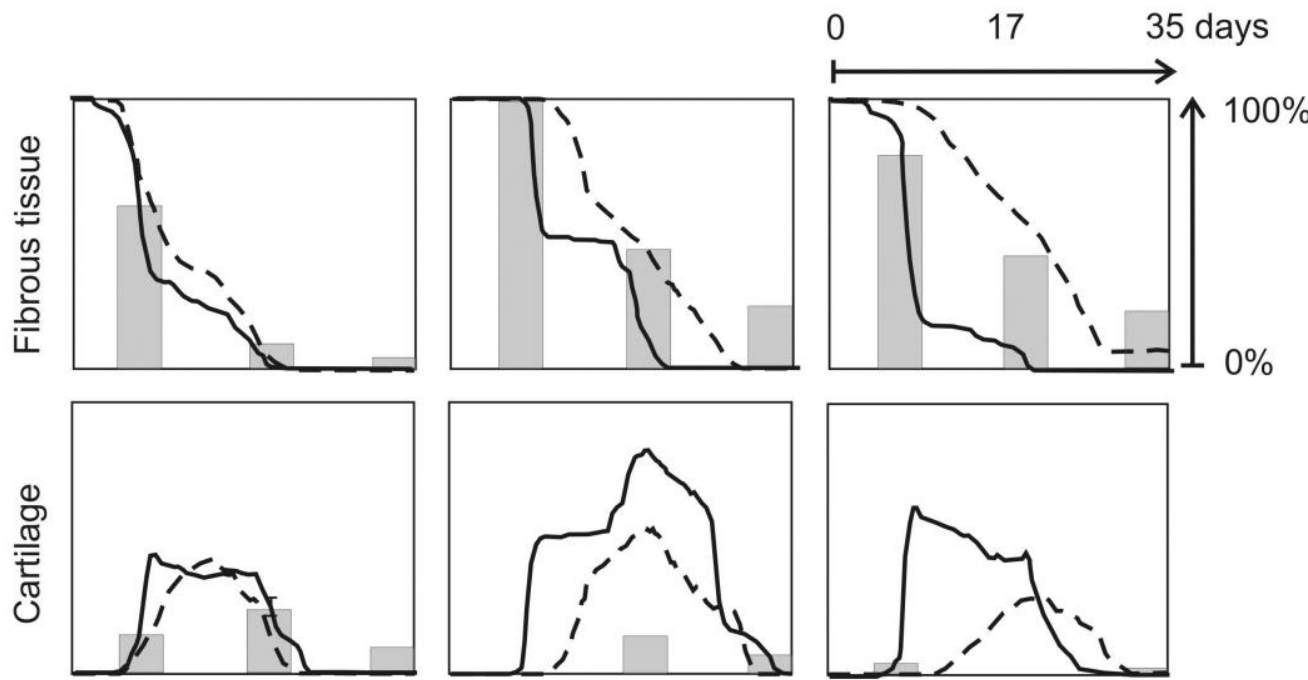

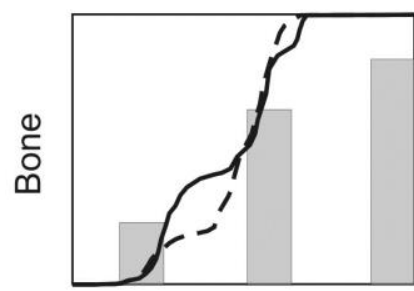

Periosteal

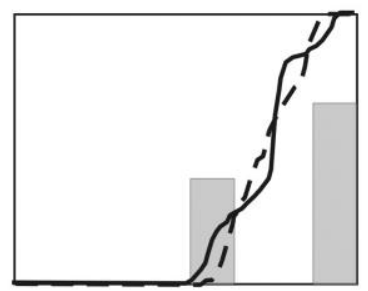

Intercortical

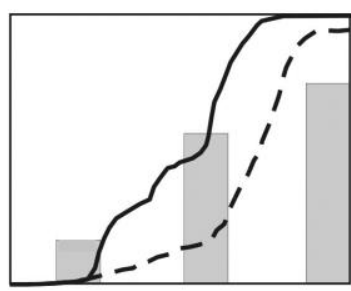

Endosteal

Figure 5. In silico and in vivo evolution of normal fracture healing in a small defect $(0.5$ $\mathrm{mm}$ ). Temporal evolution of the bone, cartilage and fibrous tissue fractions (\%) in the periosteal, intercortical and endosteal callus as predicted using the MOSAIC model of Carlier et al. (Carlier et al., 2012) and the newly developed multiscale oxygen model and as measured by Harrison et al (Harrison et al., 2003). In order to calculate the tissue fractions, the spatial images (Figure 3 ) are first binarized using tissue-specific thresholds ( 0 means that the tissue is not present, 1 means that the tissue is present in a grid cell). Subsequently, an equal weight is assigned to the different tissues, i.e. if a grid cell contains three tissues, the area of that grid cell is divided by three in the final calculations of the tissue (area) fractions.

The predictions of the MOSAIC model and the new oxygen model agree with the experimental data of Harrison et al. (Harrison et al., 2003) who determined histologically the distribution of fibrous tissue, bone and cartilage for three different zones in the fracture callus (i.e. intercortical, endosteal and periosteal) of a standardized rat femoral model (Figure 5). Both the MOSAIC model as well as the oxygen model predict the general trends in the experimental data of Harrison et al. (Harrison et al., 2003): as the healing process 
continues the bone tissue fraction and fibrous tissue fraction monotonically increase and decrease respectively whereas the cartilage tissue fraction is first produced during the soft callus phase and later replaced by bone during endochondral ossification. The amount of cartilage predicted by the MOSAIC model is different from that predicted by the oxygen model, i.e. in the endosteal and intercortical callus the oxygen model predicts more cartilage. Since the proliferation and differentiation rates are oxygen dependent in the oxygen model, also the dynamics of the MOSAIC and oxygen model are slightly different. In the latter, the chondrocytes survive better, reaching their maximal cell density which leads to an increased amount of cartilage production. Moreover, endochondral ossification starts a bit closer to the bony ends in the endosteal callus resulting in higher bone tissue fractions at earlier time points. The oxygen model does not only capture the correct dynamics of the fracture healing process at the tissue scale, it also correctly simulates the intracellular dynamics of the DII4-Notch signaling pathway which in turn determines the ingrowth of the vascular tree in the fracture callus (Figure 3G).

\subsection{Sensitivity analysis}

The results of the sensitivity analyses that were performed are summarized in Tables 4 and 5. The initial positions of the endothelial cells, provided as a figure in the supplementary material, have a small influence on the final tissue fractions (+/- 5\%). This difference is due to a different spatial filling of the blood vessels in the 2D simulated fracture callus and is in the same range as the influence of the random fibers on the simulation outcome $(+/-3 \%$ (Peiffer et al., 2011)). Both the initial conditions and the boundary conditions have little influence on the final outcome of the oxygen model (Table 3). All the variations lie in the same range as the variations due to small changes in endothelial cell position or fiber orientation. Due to 
the importance of the osteoprogenitor cells in fracture healing, we investigated the influence of a gradient or random distribution of these cells on the simulation outcome, but also these are insignificant. Similarly, the initial distribution (uniform, gradient or random) of oxygen in the fracture callus does not influence the simulation outcome.

Table 3: Overview of results of the sensitivity analysis on the initial position of the endothelial cells, the initial conditions and the boundary conditions. The tissue fractions are measured at post fracture day 35. The non-dimensionalized parameter values corresponding to the standard condition are $\tilde{m}_{f, \text { init }}=\mathbf{0 . 1}, \tilde{g}_{b c \text {,init }}=\mathbf{1}, \tilde{c}_{m \text {,init }}=\mathbf{0 . 0 2 ,} \tilde{c}_{f, \text { init }}=$ $0.01, \tilde{\boldsymbol{n}}_{\text {init }}=\mathbf{0 . 0 3 7 + 0 . 0 8 2 5} \tilde{x}, \tilde{g}_{b c, B C}=20, \tilde{c}_{m, B C}=\mathbf{0 . 0 2}$ and $\tilde{c}_{f, B C}=\mathbf{0 . 0 2}$. $\tilde{x}$ represents the non-dimensionalized coordinate on the horizontal axis in Figure 2. Rand represents a randomly chosen number between 0 and 1 . A graphical representation of the initial positions of the ECs as well as the non-dimensionalized parameter values can be found in the supplementary material. The standard condition is indicated in bold.

\begin{tabular}{|c|c|c|c|c|}
\hline \multicolumn{2}{|c|}{ Condition } & \multirow{2}{*}{$\begin{array}{c}\text { Bone } \\
100.00 \%\end{array}$} & \multirow{2}{*}{$\begin{array}{c}\text { Fibrous matrix } \\
0.00 \%\end{array}$} & \multirow{2}{*}{$\begin{array}{c}\text { Cartilage } \\
0.00 \%\end{array}$} \\
\hline standard & & & & \\
\hline EC position 1 & & $100.00 \%$ & $0.00 \%$ & $0.00 \%$ \\
\hline EC position 2 & & $99.10 \%$ & $0.00 \%$ & $0.90 \%$ \\
\hline EC position 3 & & $100.00 \%$ & $0.00 \%$ & $0.00 \%$ \\
\hline EC position 4 & & $95.12 \%$ & $0.00 \%$ & $4.88 \%$ \\
\hline EC position 5 & & $86.35 \%$ & $0.00 \%$ & $13.55 \%$ \\
\hline \multirow[t]{2}{*}{$\tilde{m}_{f, \text { init }}$} & 0.05 & $100.00 \%$ & $0.00 \%$ & $0.00 \%$ \\
\hline & 0.15 & $100.00 \%$ & $0.00 \%$ & $0.00 \%$ \\
\hline \multirow[t]{2}{*}{$\tilde{g}_{b c, \text { init }}$} & 0.5 & $100.00 \%$ & $0.00 \%$ & $0.00 \%$ \\
\hline & 1.5 & $100.00 \%$ & $0.00 \%$ & $0.00 \%$ \\
\hline \multirow[t]{4}{*}{$\tilde{c}_{m, i n i t}$} & 0.01 & $100.00 \%$ & $0.00 \%$ & $0.00 \%$ \\
\hline & 0.03 & $98.44 \%$ & $0.00 \%$ & $1.56 \%$ \\
\hline & $0.015+0.007 \tilde{x}$ & $100.00 \%$ & $0.00 \%$ & $0.00 \%$ \\
\hline & $0.015+0.01$ rand & $100.00 \%$ & $0.00 \%$ & $0.00 \%$ \\
\hline \multirow[t]{2}{*}{$\tilde{c}_{f, \text { init }}$} & 0.005 & $99.61 \%$ & $0.00 \%$ & $0.39 \%$ \\
\hline & 0.015 & $100.00 \%$ & $0.00 \%$ & $0.00 \%$ \\
\hline \multirow[t]{6}{*}{$\tilde{n}_{\text {init }}$} & $0.037+0.0625 \tilde{x}$ & $100.00 \%$ & $0.00 \%$ & $0.00 \%$ \\
\hline & $0.037+0.125 \tilde{x}$ & $100.00 \%$ & $0.00 \%$ & $0.00 \%$ \\
\hline & $0.027+0.0825 \tilde{x}$ & $92.07 \%$ & $0.00 \%$ & $7.93 \%$ \\
\hline & $0.047+0.0625 \tilde{x}$ & $100.00 \%$ & $0.00 \%$ & $0.00 \%$ \\
\hline & 0.04 uniform & $100.00 \%$ & $0.00 \%$ & $0.00 \%$ \\
\hline & $0.037+0.005$ rand & $100.00 \%$ & $0.00 \%$ & $0.00 \%$ \\
\hline$\overline{\tilde{g}_{b c, B C}}$ & 10 & $100.00 \%$ & $0.00 \%$ & $0.00 \%$ \\
\hline
\end{tabular}




\begin{tabular}{|c|c|c|c|c|}
\hline & 30 & $99.69 \%$ & $0.00 \%$ & $0.31 \%$ \\
\hline \hline$\tilde{c}_{m, B C}$ & 0.01 & $100.00 \%$ & $0.00 \%$ & $0.00 \%$ \\
\hline & 0.03 & $100.00 \%$ & $0.00 \%$ & $0.00 \%$ \\
\hline \hline$\tilde{c}_{f, B C}$ & 0.01 & $100.00 \%$ & $0.00 \%$ & $0.00 \%$ \\
\hline & 0.03 & $99.29 \%$ & $0.08 \%$ & $0 \%$ \\
\hline
\end{tabular}

Table 4: Overview of results of the sensitivity analysis on the oxygen model parameters. The tissue fractions are measured at post fracture day 35. The oxygen model parameters corresponding to the standard condition are $F_{5,1} 0.5 \%, F_{5,2} 11 \%, F_{6} 2 \%, F_{7} 0.5 \%, F_{8} 2.25 \%$, $E_{\text {hyp }, m} 2 \%, E_{\text {hyp }, c} 1.5 \%, E_{h y p, b} 4 \%, E_{\text {hyp }, f} 4.5 \%, d_{n m} 2 \%, d_{n c} 1.5 \%, d_{n b} 4 \%, d_{n f} 4.5 \%, A_{m} 4 \%, A_{c} 3 \%$, $A_{b} 8 \%, A_{f} 9 \%, F_{1} 8 \%, F_{2} 3 \%, F_{3} 8 \%$ (Table 1). The non-dimensionalized parameter values can be found in the supplementary material. The standard condition is indicated in bold.

\begin{tabular}{|c|c|c|c|c|c|}
\hline Condition & $\begin{array}{l}\text { Functional } \\
\text { form }\end{array}$ & $\begin{array}{l}\text { Oxygen } \\
\text { threshold }\end{array}$ & Bone & $\begin{array}{l}\text { Fibrous } \\
\text { matrix }\end{array}$ & $\begin{array}{l}\text { Cartilage } \\
\text { matrix }\end{array}$ \\
\hline standard & & & 100.00 & $0.00 \%$ & $0.00 \%$ \\
\hline \multirow{4}{*}{ chondrocyte cell death } & \multirow{2}{*}{$F_{5,1}$} & $0 \%$ & $100.00 \%$ & $0.00 \%$ & $0.00 \%$ \\
\hline & & $1.5 \%$ & $64.92 \%$ & $18.64 \%$ & $16.44 \%$ \\
\hline & \multirow{2}{*}{$F_{5,2}$} & $10 \%$ & $100.00 \%$ & $0.00 \%$ & $0.00 \%$ \\
\hline & & $12 \%$ & $100.00 \%$ & $0.00 \%$ & $0.00 \%$ \\
\hline \multirow{2}{*}{ osteoblast cell death } & \multirow{2}{*}{$F_{6}$} & $1 \%$ & $99.92 \%$ & $0.00 \%$ & $0.08 \%$ \\
\hline & & $3 \%$ & $100.00 \%$ & $0.00 \%$ & $0.00 \%$ \\
\hline \multirow{2}{*}{ MSC cell death } & \multirow[t]{2}{*}{$F_{7}$} & $0 \%$ & $100.00 \%$ & $0.00 \%$ & $0.00 \%$ \\
\hline & & $1.5 \%$ & $100.00 \%$ & $0.00 \%$ & $0.00 \%$ \\
\hline \multirow{2}{*}{ fibroblast cell death } & \multirow[t]{2}{*}{$F_{8}$} & $1.25 \%$ & $100.00 \%$ & $0.00 \%$ & $0.00 \%$ \\
\hline & & $3.25 \%$ & $99.84 \%$ & $0.00 \%$ & $0.16 \%$ \\
\hline \multirow{2}{*}{$\begin{array}{c}\text { hypoxia-dependent } g_{v} \text { production } \\
\text { by MSCs }\end{array}$} & \multirow{2}{*}{$E_{h y p, m}$} & $1 \%$ & $100.00 \%$ & $0.00 \%$ & $0.00 \%$ \\
\hline & & $3 \%$ & $100.00 \%$ & $0.00 \%$ & $0.00 \%$ \\
\hline \multirow{2}{*}{$\begin{array}{l}\text { hypoxia-dependent } g_{v} \text { production } \\
\text { by chondrocytes }\end{array}$} & \multirow{2}{*}{$E_{\text {hyp }, c}$} & $0.5 \%$ & $100.00 \%$ & $0.00 \%$ & $0.00 \%$ \\
\hline & & $2.5 \%$ & $100.00 \%$ & $0.00 \%$ & $0.00 \%$ \\
\hline \multirow{2}{*}{$\begin{array}{l}\text { hypoxia-dependent } g_{v} \text { production } \\
\text { by osteoblasts }\end{array}$} & \multirow{2}{*}{$E_{h y p, b}$} & $3 \%$ & $100.00 \%$ & $0.00 \%$ & $0.00 \%$ \\
\hline & & $5 \%$ & $100.00 \%$ & $0.00 \%$ & $0.00 \%$ \\
\hline \multirow{2}{*}{$\begin{array}{l}\text { hypoxia-dependent } g_{\mathrm{v}} \text { production } \\
\text { by fibroblasts }\end{array}$} & \multirow{2}{*}{$E_{\text {hyp, } f}$} & $3.5 \%$ & $100.00 \%$ & $0.00 \%$ & $0.00 \%$ \\
\hline & & $5.5 \%$ & $98.63 \%$ & $0.00 \%$ & $1.37 \%$ \\
\hline \multirow{2}{*}{$\begin{array}{l}\text { half maximal oxygen consumption } \\
\text { rate of MSCs }\end{array}$} & \multirow{2}{*}{$d_{n m}$} & $1 \%$ & $100.00 \%$ & $0.00 \%$ & $0.00 \%$ \\
\hline & & $3 \%$ & $100.00 \%$ & $0.00 \%$ & $0.00 \%$ \\
\hline \multirow{2}{*}{$\begin{array}{l}\text { half maximal oxygen consumption } \\
\text { rate of chondrocytes }\end{array}$} & \multirow[t]{2}{*}{$d_{n c}$} & $0.5 \%$ & $98.44 \%$ & $0.00 \%$ & $1.56 \%$ \\
\hline & & $2.5 \%$ & $100.00 \%$ & $0.00 \%$ & $0.00 \%$ \\
\hline \multirow{2}{*}{$\begin{array}{c}\text { half maximal oxygen } \\
\text { consumption rate of osteoblasts }\end{array}$} & \multirow[t]{2}{*}{$d_{n b}$} & $3 \%$ & $100.00 \%$ & $0.00 \%$ & $0.00 \%$ \\
\hline & & $5 \%$ & $98.67 \%$ & $0.00 \%$ & $1.33 \%$ \\
\hline \multirow{2}{*}{$\begin{array}{c}\text { half maximal oxygen } \\
\text { consumption rate of fibroblasts }\end{array}$} & \multirow[t]{2}{*}{$d_{n f}$} & $3.5 \%$ & $100.00 \%$ & $0.00 \%$ & $0.00 \%$ \\
\hline & & $5.5 \%$ & $100.00 \%$ & $0.00 \%$ & $0.00 \%$ \\
\hline
\end{tabular}




\begin{tabular}{|c|c|c|c|c|c|}
\hline \multirow{2}{*}{ MSC proliferation } & \multirow{2}{*}{$A_{m}$} & $3 \%$ & $100.00 \%$ & $0.00 \%$ & $0.00 \%$ \\
\cline { 3 - 5 } & & $5 \%$ & $100.00 \%$ & $0.00 \%$ & $0.00 \%$ \\
\hline \hline \multirow{2}{*}{ chondrocyte proliferation } & \multirow{2}{*}{$A_{c}$} & $2 \%$ & $82.34 \%$ & $11.41 \%$ & $6.25 \%$ \\
\cline { 3 - 5 } & & $4 \%$ & $98.48 \%$ & $0.00 \%$ & $1.52 \%$ \\
\hline \multirow{2}{*}{ osteoblast proliferation } & \multirow{2}{*}{$A_{b}$} & $7 \%$ & $100.00 \%$ & $0.00 \%$ & $0.00 \%$ \\
\cline { 3 - 6 } & & $9 \%$ & $100.00 \%$ & $0.00 \%$ & $0.00 \%$ \\
\hline \hline \multirow{2}{*}{ fibroblast proliferation } & \multirow{2}{*}{$A_{f}$} & $8 \%$ & $100.00 \%$ & $0.00 \%$ & $0.00 \%$ \\
\cline { 3 - 6 } & & $10 \%$ & $100.00 \%$ & $0.00 \%$ & $0.00 \%$ \\
\hline \hline \multirow{2}{*}{ osteogenic differentiation } & \multirow{2}{*}{$F_{1}$} & $7 \%$ & $100.00 \%$ & $0.00 \%$ & $0.00 \%$ \\
\cline { 3 - 6 } & & $9 \%$ & $100.00 \%$ & $0.00 \%$ & $0.00 \%$ \\
\hline \multirow{2}{*}{ chondrogenic differentiation } & \multirow{2}{*}{$F_{2}$} & $2 \%$ & $99.96 \%$ & $0.04 \%$ & $0.00 \%$ \\
\cline { 3 - 6 } & & $4 \%$ & $100.00 \%$ & $0.00 \%$ & $0.00 \%$ \\
\hline \hline \multirow{2}{*}{ endochondral ossification } & \multirow{2}{*}{$F_{3}$} & $7 \%$ & $100.00 \%$ & $0.00 \%$ & $0.00 \%$ \\
\cline { 3 - 6 } & & $9 \%$ & $97.58 \%$ & $0.00 \%$ & $2.42 \%$ \\
\hline
\end{tabular}

Also the parameters of the different oxygen dependent processes in the fracture healing model have little influence on the final outcome (Table 4); except for the functional forms $A_{c}$ and $F_{5,1}$ which will be discussed below (Table 4). Again all the variations in model outcome lie in the same range as the variations due to small changes in endothelial cell position or fiber orientation. 


\subsection{Impaired fracture healing}

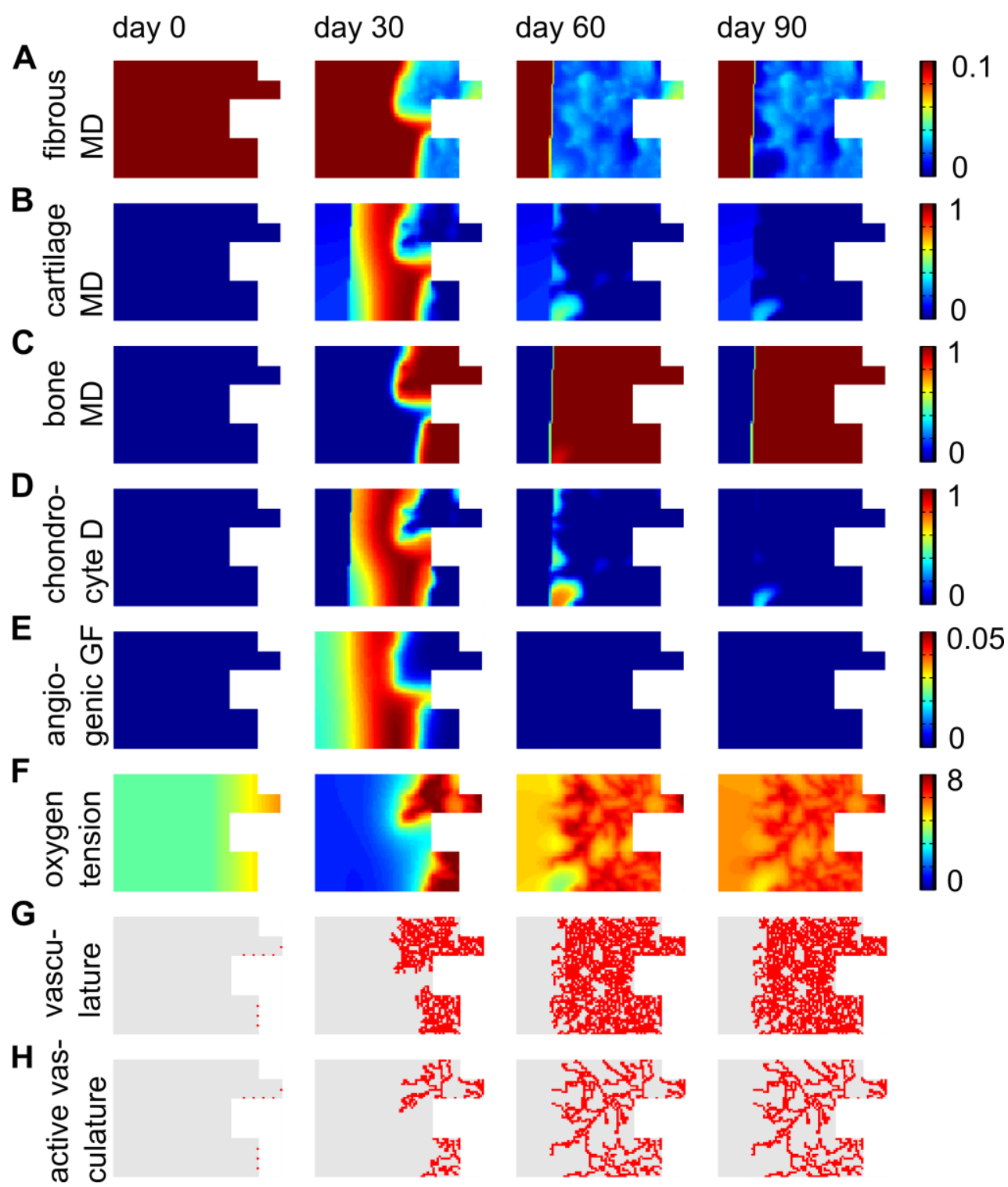

Figure 6: The spatiotemporal evolution of $(A)$ fibrous tissue matrix density (MD) $(x 0.1$ $\mathrm{g} / \mathrm{ml}),(B)$ cartilage matrix density $(\times 0.1 \mathrm{~g} / \mathrm{ml}),(C)$ bone matrix density $(\times 0.1 \mathrm{~g} / \mathrm{ml}),(D)$ chondrocyte density ( $x 10^{6}$ cells $/ \mathrm{ml}$ ), (E) angiogenic growth factor concentration (GF) (x $100 \mathrm{ng} / \mathrm{ml}$ ), (F) oxygen tension (x 1\%), (G) vasculature and $(H)$ active vasculature during impaired fracture healing in a large defect $(3 \mathrm{~mm})$ as predicted by the oxygen model.

After corroboration, the oxygen model was used to predict the spatiotemporal evolution of the fracture healing process in a $3 \mathrm{~mm}$ defect (Figure 6). Similar to normal bone healing, osteoprogenitor cells initially populate the fracture callus. Around the bony ends some direct bone formation can be found and further away from the bony ends a cartilage template is 
formed by the chondrocytes (Figure 6B-D). As the chondrocytes become hypertrophic and produce the angiogenic growth factor $\left(g_{v}\right)$ (Figure 6E), the blood vessels grow into the cartilage callus leading to the gradual replacement of the cartilage template by bone (Figure 6B-C). In the central region of the fracture callus, however, all the cells die due to the harsh hypoxic conditions (Figure 6D-F, day 30). Consequently, the angiogenic growth factor $\left(g_{v}\right)$, which is the major stimulus for vascular growth and as such endochondral ossification, is no longer produced and the bone healing process stops (Figure 6E). Remark that in the oxygen model, although all the cells have died in the central fracture zone, the blood vessels continue to deliver oxygen which is no longer consumed and eventually floods the entire callus (Figure 6F). Between 58 and 90 days all signs of the healing process are completely absent, classifying this fracture as a non-union (Roberts and Rosenbaum, 2012;Marsh, 1998).
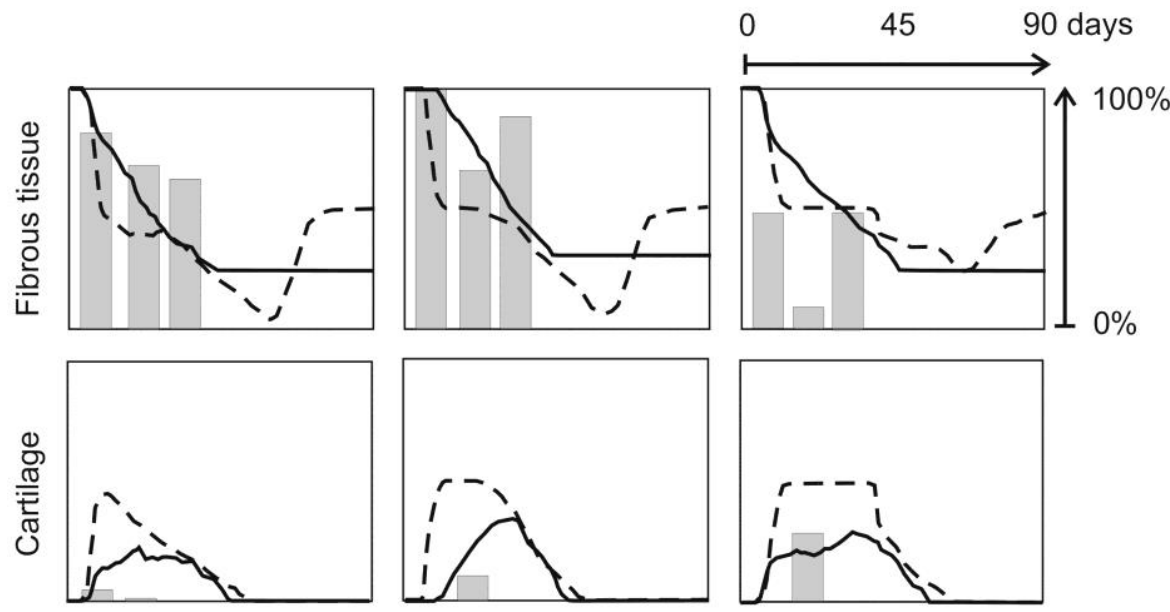

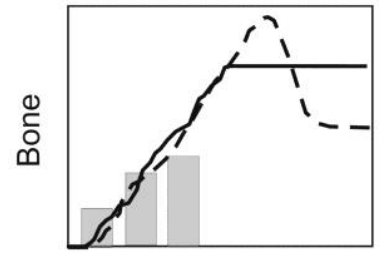

Periosteal

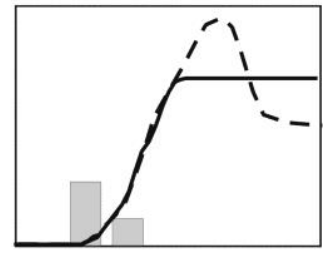

Intercortical

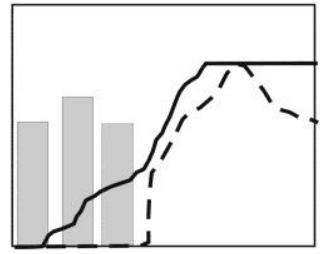

Endosteal

$$
\text { - Oxygen model } \quad-- \text { MOSAIC model } \quad \text { Experimental data }
$$

Figure 7. In silico and in vivo evolution of impaired fracture healing in a large defect (3 $\mathrm{mm}$ ). Temporal evolution of the bone, cartilage and fibrous tissue fractions (\%) in the periosteal, intercortical and endosteal callus as predicted using the MOSAIC model of Carlier et al. (Carlier et al., 2012) and the newly developed multiscale oxygen model and as measured by Harrison et al (Harrison et al., 2003). 
Figure 7 compares the predicted and measured tissue fractions for the standardized rat femoral fracture with a $3 \mathrm{~mm}$ gap size. Firstly, it can be seen that both models capture the cartilage dynamics where the cartilage template is first produced and later replaced by bone. Secondly, both the simulation results of the oxygen model as well as the experimental data predict abundant fibrous tissue in the fracture callus after 90 days, clearly indicating a clinical non-union. In the MOSAIC model, however, the endochondral ossification process continues resulting in a union after 90 days (Figure $8 \mathrm{C}$ ). Remark that the MOSAIC model predicts much more cartilage formation than the oxygen model (Figure $8 \mathrm{~B}$ ) and a very heterogeneous pattern of fibrous tissue formation (Figure $8 \mathrm{~A}$, day 60 ). The former can be explained by the absence of cell death in the MOSAIC model whereas the latter is a result of the combined removal and production of fibrous tissue (Equation 5). In the MOSAIC model, the fibroblasts continue to survive and to produce fibrous tissue resulting in a bony union with a large fibrous component.

A closer comparison of results of the oxygen model and experimentally measured data shows that cartilage is formed later in the experimental model than predicted by the oxygen model. In all the fracture regions and for all the time points the latter also predicts more cartilage except for day 21 in the endosteal callus. There is more bone present in the experimental model at day 7 and 21 and this additional bone is gradually being resorbed and remodeled to form a rounded osseous cap over the medullary cavities (Harrison et al., 2003). The latter is not included in the oxygen model, explaining why after a continued formation of bone up to 58 days the bone tissue fraction stays constant (Figures 6C, 7). From the above results we might conclude that even though the timing of the oxygen model does not entirely correspond to the experimentally observed dynamics, and the simulation results 
overestimate the amount of bone formation, the oxygen model it is able to correctly predict whether the bone fracture will result in a clinical union or non-union.

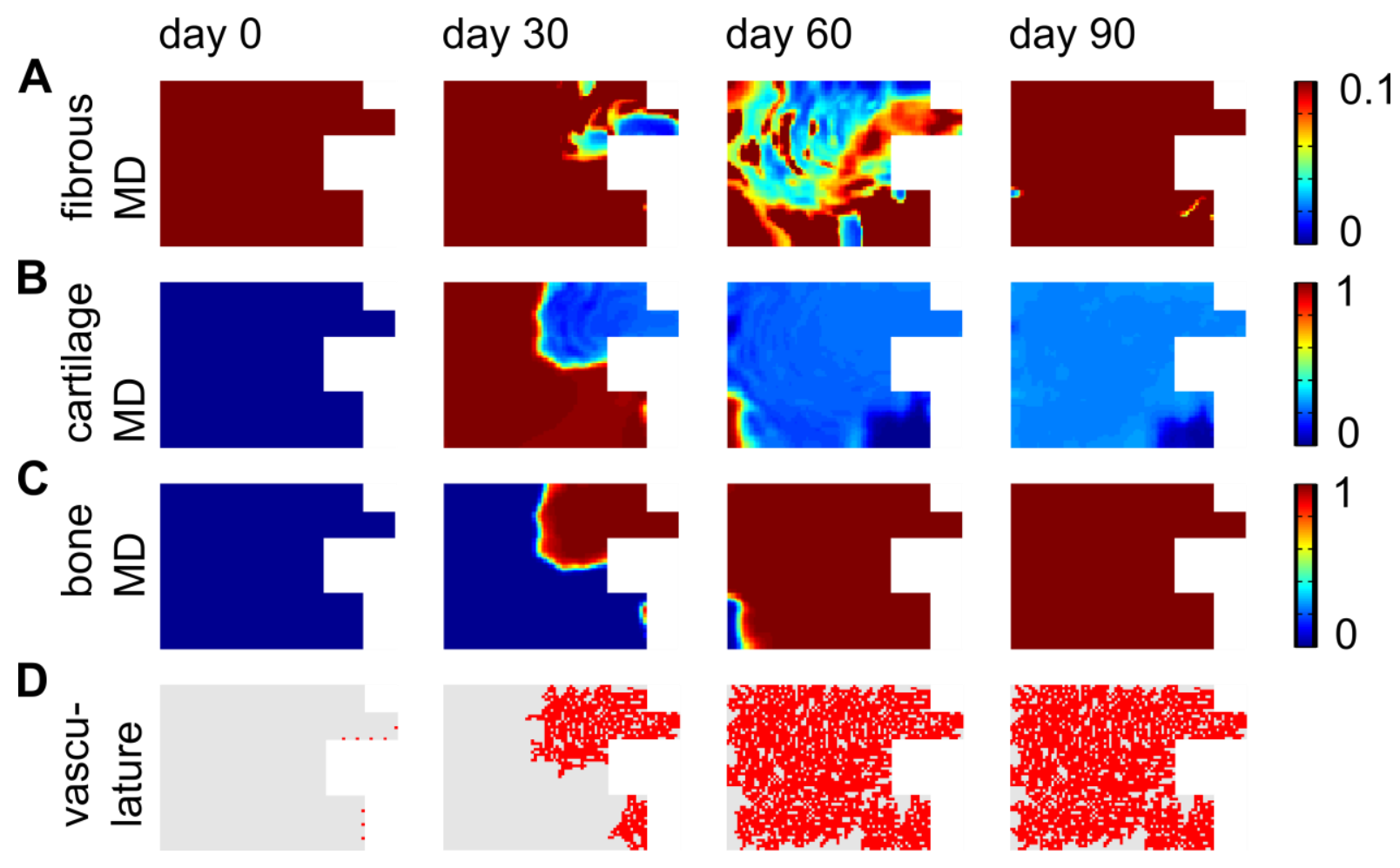

Figure 8: The spatiotemporal evolution of $(A)$ fibrous tissue matrix density (MD) (x 0.1 $\mathrm{g} / \mathrm{ml}),(B)$ cartilage matrix density $(x 0.1 \mathrm{~g} / \mathrm{ml})$, (C) bone matrix density $(\times 0.1 \mathrm{~g} / \mathrm{ml})$ and (D) vasculature during impaired fracture healing in a large defect $(3 \mathrm{~mm})$ as predicted by the MOSAIC model. 


\section{Discussion}

This study has presented a novel multiscale model of bone fracture healing including a detailed description of the regulatory properties of oxygen on the behavior of skeletal cells occurring during normal and impaired fracture healing. A rigorous literature screening was performed in order to ensure that the oxygen dependency of a number of cellular processes, i.e. osteogenic and chondrogenic differentiation, cell proliferation, cell death, oxygen consumption and the hypoxia-dependent production of angiogenic growth factors, was informed by state-of-the-art experimental knowledge. Furthermore, an extensive sensitivity analysis on the newly introduced oxygen thresholds demonstrated the robustness of the oxygen model with respect to changes in the oxygen related parameter values. Not only is the novel oxygen model able to simulate the spatiotemporal evolution of the bone regeneration process in both normal as well as impaired healing cases, it captures the intracellular (in terms of Dll4-Notch) and cellular dynamics of the developing vascular structures as well. We have also shown that the results of the oxygen model are in accordance with experimental reports for small and large defect sizes. Indeed, when the oxygen model was applied to a critical size defect, it correctly predicted the establishment of a clinical non-union which could be explained by the imbalance between the rate of ingrowth of new blood vessels (and thus oxygen delivery) and the oxygen consumption of the cells, leading to hypoxic conditions and cell death in the central region of the fracture callus. As such, the accurate description of the oxygen dependent behavior of skeletal cells, which is the essence of the novel oxygen model, allows us to investigate the spatiotemporal oxygen patterns that are an important determinant of fracture healing. 
Except for some interesting cases (EC position $5, \tilde{n}_{\text {init }} A_{c}, F_{5,1}$ ) that will be discussed below, the results of the extensive sensitivity analysis show that the oxygen model is robust to variations in initial and boundary conditions, as well as to the newly defined parameters that capture oxygen-dependent cellular processes. Based on Table 4 we can also state that chondrocyte death in pathologically high oxygen environments $\left(F_{5,2}\right)$ has no influence on the model outcome. Even more, bearing in mind that the oxygen tensions do not exceed $12 \%$ in the model simulations and that we are mainly interested in the lack of oxygen due to a mismatch of oxygen diffusion, delivery and consumption, the oxygen model could be simplified in the future by removing the term $F_{5,2}$.

Since the initial position of the ECs influences the final bone tissue fraction with $+/-5 \%$, deviations of more than $5 \%$ with respect to the results of the standard case can be considered significant. Significantly different results were obtained for four cases: EC position 5 , initial oxygen distribution $\left(\tilde{n}_{\text {init }}\right)$, chondrocyte proliferation $\left(A_{c}\right)$ and chondrocyte death in low oxygen $\left(F_{5,1}\right)$ (Tables 4 and 5 ). In order to gain more understanding in the complex non-linear dynamics of the oxygen model, the mechanisms underlying these significant deviations were investigated further and are discussed in more detail below.

In the first two cases (EC position $5, \tilde{n}_{\text {init }}$ ) we noticed that the tip cells would migrate slower or would not migrate at all into the fracture callus although favorable gradients for haptoand chemotaxis were available. This resulted in a delay of the process without affecting the main mechanisms underlying the events of fracture healing. Consequently, given more time for vessel ingrowth, these cases would yield similar tissue fractions as the standard case. The slower ingrowth of the tip cells appeared to be attributed to the strong neighboring DII4Notch signaling. In order to investigate this further we tested whether an increase in the distance over which the ECs can sense the angiogenic growth factor (e.g. through filopodia) 
would overcome this local artifact. Indeed, using an average angiogenic growth factor concentration surrounding the EC instead of the localized angiogenic growth factor concentration as an input for the intracellular module resulted in normal tip cell movement and formation of the vasculature (results not shown). Future work should focus on a more detailed description of filopodia and the way by which these cellular protrusions sense and interact with their environment in order to improve the link between the tissue scale and the intracellular scale.

In the third case, where the optimal oxygen tension for chondrocyte proliferation is set at $2 \%\left(A_{c}\right.$, Table 4$)$, the chondrocytes preferentially grow further away from the bony ends than in the standard case (where the optimal oxygen tension for chondrocyte proliferation is 3\%). This results in a lower cell density mainly in the endosteal callus which consequently leads to a flooding of the endosteal callus with oxygen. Indeed, in the oxygen model the newly formed vasculature continuously acts as a source of oxygen, independently of the presence of surrounding cells. Hence, oxygen starts to accumulate and diffuse from the regions where due to lower cell density also the oxygen consumption is reduced. The flooding of the endosteal callus pushes the chondrocytes even further away since they preferentially proliferate in low oxygen environments resulting in the absence of endochondral ossification and a limited amount of bone formation in the endosteal callus and consequently also an overall lower bone tissue fraction $\left(A_{c}\right.$, oxygen threshold $2 \%$, Table 4$)$. The fourth case can be explained by the same mechanism since also an increase in the oxygen parameter value describing chondrocyte cell death $\left(F_{5,1}\right.$, Table 4$)$ will result in a lower cell density, eventually leading to the flooding of the callus and a lower amount of bone formation. Clearly, the two cases discussed above pinpoint a limitation of the current oxygen model, i.e. the delivery of 
oxygen is independent of the surrounding cell density leading to unexpected results in cases of low cell density. This model limitation is discussed in more detail below.

Figures 3, S2 and 5 illustrate that both the MOSAIC model as well as the oxygen model capture equally well the spatiotemporal dynamics of the fracture healing process. However, a more in depth comparison between the models and the in vivo observations reveals some interesting differences. In the oxygen model a quicker resorption of the fibrous tissue matrix is seen than in the MOSAIC model. This is due to the larger extent of endochondral ossification (especially in the endosteal callus) in the oxygen model. Remark that only endochondral ossification leads to resorption of the fibrous tissue matrix in contrast to intramembranous bone formation (equations 5-7). The increase in the amount of endochondral ossification in the oxygen model is linked to a second difference with the MOSAIC model, i.e. a more homogeneous distribution of cartilage throughout the fracture callus. This discrepancy can be explained by the way differentiation is captured in the two models. In the MOSAIC model, an osteogenic and chondrogenic growth factor determine the lineage to which the MSCs will differentiate whereas in the oxygen model the local oxygen tension will steer the differentiation process by mediating the effect of the osteochondrogenic growth factor. Consequently, in the MOSAIC model the spatial distribution of the cartilage template is mainly determined by the location of the boundary conditions (applied periosteally) resulting in less cartilage formation in the endosteal callus. In the oxygen model, however, the local oxygen tensions will determine the spatiotemporal cartilage distribution, allowing cartilage formation in the entire fracture callus. As such the MOSAIC model predicts better the in vivo data for fibrous tissue and cartilage than the oxygen model. Note that in the oxygen model the inner part of the external callus is first 
resorbed and replaced by bone and then the upper left corner is calcified (Figure 3, day 21) (a similar pattern is predicted by the MOSAIC model, see additional Figure S2). This atypical resorption pattern is predicted by the models due to the specific pattern of (active) vasculature formation leading to locally high oxygen tensions and endochondral ossification. A limitation of both the MOSAIC and the oxygen model is that all the progenitor cells can differentiate towards both the chondrogenic and osteogenic lineage. In reality, however, it was shown that the progenitors from the endosteal callus can only differentiate towards the osteogenic lineage, resulting in the absence of cartilage in the endosteal callus (Colnot, 2009). Progenitor cells from the periosteum do have the capability to differentiate in both lineages, explaining why endochondral ossification mainly occurs in the periosteal callus (Colnot, 2009). Consequently, the current simplification of the models leads to an overestimation of the amount and the location of the cartilage matrix (see Figure 5). In future versions of the model, an additional variable can be introduced to discriminate between periosteum-derived and bone marrow-derived progenitor cells. In conclusion, we can state that the new oxygen model performs better for the prediction of bone tissue fractions (in particular in the endosteal callus) when comparing the two models to the in vivo data of Harrison et al. (Harrison et al., 2003). Given the fact that in terms of functional outcome bone density is the most important variable, this is an improvement of the new model with respect to the previous one. At the same time, future work should focus on implementing additional variables to discriminate between periosteum-derived and bone marrow-derived progenitor cells so that also the fibrous tissue fractions and cartilage fractions are correctly captured. However, the main goal of extending the MOSAIC model with an accurate description of the influence of oxygen on fracture healing was to increase its application possibilities and not to improve its predictive capacities. Indeed, the oxygen 
model is able to capture the formation of a non-union, an application which is clinically very relevant (see below).

Figures 6 and 7 show that the oxygen model is able to predict the formation of a non-union. The predicted bone tissue fractions are however larger than the experimentally observed ones by Harrison et al. (Harrison et al., 2003) (Figure 7). This could amongst others be due to the fact that the same (favorable) initial conditions as for normal fracture healing (gap size $0.5 \mathrm{~mm}$ ) were kept in the entire callus area of the critically sized defect. Experimental evidence indicates that the biological potential (e.g. the amount of cells and growth factors present) might be greatly reduced in critically sized defects (Stevens, 2008;Bruder and Fox, 1999). Moreover, no data exist on the exact value of the oxygen tension in the central region of large defects. In the simulation of the critically sized defect the oxygen tension was kept uniform and equal to $3.7 \%$ in the central callus area, while the same gradient as for the standard case was applied near the bony ends (Figure 6)). In reality the oxygen tension in the central region of large defects may be even lower than $3.7 \%$. The MOSAIC model, which does not model the oxygen dependency of the fracture healing processes, fails to predict a non-union in the case of a large defect (Figure 8C). Indeed, due to the absence of amongst others oxygen consumption and cell death, the chondrocytes continue to survive and populate the entire fracture callus (Figures 6D, 8D). Consequently, a cartilage template is laid down in the entire callus, and not only close to the bony ends as is the case in the oxygen model (Figures 6B, 8B), allowing the endochondral ossification process to proceed until a clinical union is reached (Figure $8 \mathrm{C}$ ). Moreover, due to the absence of (fibroblastic) cell death, the fibroblasts continue to survive and proliferate, finally resulting in a bony union containing a large fibrous component as well. This can be seen in Figures 7 and $8 \mathrm{~A}$ where the 
fibrous tissue fraction starts to increase after 60 days, explaining why the bone tissue fraction does not reach $100 \%$ after 90 days although a bony union is reached. The comparison of the results of the MOSAIC model and oxygen model in large defect sizes nicely illustrates the importance of a rigorous description of the influence of oxygen: due to the imbalance between oxygen consumption and oxygen delivery through (active) revascularization and (passive) diffusion hypoxic conditions arise in the central fracture zone leading to cell death and eventually the formation of a non-union. It appears that a timely delivery of oxygen is a key factor in successfully bridging the critical size defect. Moreover, simple passive diffusion is not sufficient to supply the entire fracture callus with oxygen due to the consumption of oxygen by cells. Consequently, a timely vascularization of the fracture callus is a prerequisite for successful fracture healing. Therefore we suggest that treatments should not only focus on the injection of stem cells (Patterson et al., 2008) or growth factors (Dimitriou et al., 2011;Lissenberg-Thunnissen et al., 2011) but also, and more importantly, on a timely vascularization of the critical defect. Remark that the current boundary conditions of the endothelial cells (Figure 2) neglect the contribution of the overlying soft tissues to the revascularization of the fracture callus. Experimental studies have shown, however, that blood vessels can originate from the overlying muscle (Masquelet, 2003; Harry et al., 2009). In the future we will use the presented oxygen model for a more in depth investigation of the influence of the initial conditions, boundary conditions and oxygen patterns on the amount of bone formation in critically sized defects and possible therapies thereof.

This study has addressed some, but not all of the limitations of the previously published MOSAIC model. A first limitation is that the current oxygen model allows "self-anastomosis", 
meaning that the tip cell of a newly forming branch can fuse with this branch resulting in an active "loop". This is for example apparent on day 28 in Figure 3F where an active branch appears, extending into the cortical callus, which did not anastomose with another branch or the borders of the geometrical domain. This implementation was chosen over a more phenomenological one where an arbitrary number of endothelial cells should be contained in a vascular loop or where only connections with other vascular branches can be made in order to render it functional. Remark as well that in Figure 3E some endothelial cells seem to be connected, implying a vascular loop that is not represented in Figure 3F. This is a limitation of the graphical representation of the endothelial cells, which resolution is defined by the grid cell size $(25 \mu \mathrm{m})$. Tip cell movement is however implemented in a lattice freeway, meaning also that the actual position of the endothelial cells is not captured in figure 3E and 3F. In order to have an anastomosis in the oxygen model the tip cell needs to move into the grid cell representing a neighboring endothelial cell but the graphical representation cannot capture this (Peiffer et al., 2011). As such, the endothelial cells seem to be touching whereas in reality they are not. Only the endothelial cells that are part of the active vasculature truly form an interconnected network that allows blood flow and the supply of nutrients and oxygen.

A second limitation concerns the quantitative determination of the parameter values that describe the oxygen dependent processes. Although care was taken to choose the oxygen threshold values based on a rigorous literature study, the reported values are not always the same from one study to the other which may be related to differences in species or cell source (human MSCs (Holzwarth et al., 2010;Grayson et al., 2007), rat MSCs (Lennon et al., 2001), porcine MSCs (Meyer et al., 2010), adipose derived MSCs (Malladi et al., 2006; Xu et 
al., 2007)) and set-up (1\% oxygen (Holzwarth et al., 2010), 2\% (Xu et al., 2007;Malladi et al., 2006), 5\% (Hirao et al., 2006; Lennon et al., 2001;Meyer et al., 2010;Merceron et al., 2010)). Even so, all studies referenced above agree on the profound effects of oxygen on the behavior of skeletal cells, e.g. low oxygen tensions favor chondrogenic differentiation over osteogenic differentiation. We have tried to determine the relative order of oxygen dependent processes as accurate as possible. Moreover, the sensitivity analysis shows that the oxygen model is robust with respect to most variations in oxygen parameter values.

The value of the diffusion coefficient of oxygen in regenerating tissues, consisting of cartilage, fibrous matrix and bony matrix, is unknown. Values have been reported in literature for oxygen diffusion in the following media: $3 \times 10^{-9} \mathrm{~m}^{2} / \mathrm{s}$ in water (Malda et al., 2004b), $1.5 \times 10^{-9} \mathrm{~m}^{2} / \mathrm{s}$ in cartilage (Malda et al., 2004b), $3.1 \times 10^{-10} \mathrm{~m}^{2} / \mathrm{s}$ in fibrin (Demol et al., 2011), $4 \times 10^{-12} \mathrm{~m}^{2} / \mathrm{s}$ in tissue (MACDOUGA.JD and Mccabe, 1967), and $3 \times 10^{-10} \mathrm{~m}^{2} / \mathrm{s}$ through cells (Rumsey et al., 1990). These values are clearly higher than the diffusion coefficient of oxygen used in the presented mathematical model, i.e. $2 \times 10^{-12} \mathrm{~m}^{2} / \mathrm{s}$ (Peiffer et al., 2011). With this value the predicted oxygen profile nicely matches the one measured by Epari et al. (Epari et al., 2008) (Figure 4). Even more, simulations with higher oxygen diffusion coefficients $(x 10, \times 100)$ failed to correctly capture one of the major events of fracture healing, namely the replacement of the cartilage template by bone through endochondral ossification since the abundance of oxygen results in direct bone formation in the entire callus (results not shown). Clearly, these findings of the oxygen model raise some interesting questions regarding the mass transport properties of the fracture callus and encourage the development of new measuring techniques to accurately measure the actual 
in vivo diffusion coefficient of oxygen in different tissues arising during the bone fracture healing process.

A third limitation of the presented oxygen model was pinpointed by the sensitivity analysis, namely the mathematical treatment of oxygen release by blood vessels. In the current model, every endothelial cell that is part of a loop will start to deliver oxygen at a constant rate, regardless of the oxygen demand from the surrounding cells. In other words, in our model only changes in the vascular network (morphological adjustments) allow for alterations in the nutrient delivery since the model does not consider functional adaptations of the vasculature (i.e. the oxygen delivery is constant). This simplification results in the somewhat unexpected flooding of oxygen in regions characterized by low cell density. In reality other mechanisms, not accounted for in the model, may regulate vessel functionality such as vessel diameter and blood flow rate. Indeed, Hansen-Algenstaedt et al. used intravital microscopic techniques to visualize and quantify the process of vessel formation and microvascular function during bone repair (Hansen-Algenstaedt et al., 2006). They report that the development of the hematoma is accompanied by a significant increase in microvascular permeability and blood flow rate (Hansen-Algenstaedt et al., 2006). As the healing proceeds, the permeability (functional adaptation) decreases while the vascular density (morphological adaptation) increases (Hansen-Algenstaedt et al., 2006). More detailed computational models of blood flow in developing capillary structures have been reported (McDougall et al., 2002;Ji et al., 2006; Liu et al., 2011). In the future these models could be coupled to the oxygen model in order to get a more comprehensive understanding of the biological processes at hand. 
In the oxygen model, the biological cues in the fracture callus (i.e. growth factors and oxygen) will determine the course of the differentiation process of the MSCs. We assume implicitly that the mechanical environment of the fracture callus is permissive, meaning among others that the fracture is sufficiently stabilized to allow bone formation (either through intramembranous or endochondral ossification). As such, we do not consider any mechanoregulatory signals in the presented oxygen model. Previous work has shown that various models, based on very distinct (such as mechanical) cues can capture the gross tissue formation patterns of normal fracture healing, such as intramembranous ossification near the bony ends and endochondral ossification throughout the rest of the fracture callus (for a review see e.g. (Geris et al., 2009)). Prendergast et al. proposed for example a mechanoregulation model where maximal distortional strain and relative fluid velocity constitute the differentiation stimulus (Prendergast et al., 1997). Burke et al. formulated a model where MSC differentiation is regulated by substrate stiffness and oxygen tension (Burke and Kelly, 2012). Both models were able to capture the spatiotemporal evolution of the cartilage matrix, fibrous tissue and bone during normal fracture healing (Lacroix and Prendergast, 2002;Burke and Kelly, 2012; Isaksson et al., 2006). The formation of a non-union in a large defect was correctly predicted by Chen et al. who used a fuzzy logic algorithm to show that even in suitable mechanical conditions, inadequate nutrient supply would lead to the formation of a non-union (Chen et al., 2009). Another type of non-union, i.e. an atrophic non-union, was correctly captured by the bioregulatory model of Geris et al. (Geris et al., 2010). They further conclude that a combined presence of blood vessels, growth factors and precursor cells is crucial for successful healing of these problematic fractures.

Clearly, the type of mechanisms incorporated in a mathematical model of fracture healing will be determined by the scientific question at hand and/or by the in vivo model that is 
being studied. In the current study we wanted to explore the complex interplay of oxygen, angiogenesis and fracture healing in a mechanistic and experimentally-informed manner since we believe that (the absence of) oxygen is an important determinant for the occurrence of fracture non-unions in critical size defects. For the standardized femoral rat fracture model of Harrison et al. (Harrison et al., 2003) that was used here for validation we believe that oxygen is likely to be a more important determinant for the formation of nonunion in case of a $3 \mathrm{~mm}$ gap size than e.g. (excessive) mechanical loading. Although a numerical model (such as a finite element model) would be needed to calculate in detail the distribution of mechanical cues within the callus, we used a simple spring model to estimate the interfragmentary strains and conclude that for this animal model excessive loading did not play a role in the formation of the non-union (see supplementary material for more details).

\section{Conclusion}

In this study we have incorporated a rigorous description of the influence of oxygen on the fracture healing process in a previously developed multiscale model of bone regeneration. We have shown that the improved oxygen model predicts the spatiotemporal fractions of bone, cartilage and fibrous tissue in accordance with previously published experimental and in silico results. An extensive sensitivity analysis on the newly introduced oxygen thresholds demonstrated the robustness of the oxygen model. Interestingly, when the oxygen model was applied to a critical size defect, it correctly predicted the establishment of a clinical nonunion which was due to the slow ingrowth of new blood vessels leading to hypoxic conditions and cell death in the central region of the fracture callus. As such, the oxygen model can be used to unravel the complex interplay of oxygen delivery, diffusion and 
consumption with the several healing steps that all occur at specific oxygen tensions during bone fracture healing. Moreover, the spatiotemporal oxygen patterns that arise from this complex interplay potentially contribute to a more fundamental understanding of the occurrence of fracture non-unions and can lead to the design of possible therapies thereof.

\section{Acknowledgements}

Aurélie Carlier is a PhD fellow of the Research Foundation Flanders (FWO-Vlaanderen). Nick van Gastel is funded by a Concerted Research Activities Belgium Grant (GOA/13/016). The work is part of Prometheus, the Leuven Research and Development Division of Skeletal Tissue Engineering of the Katholieke Universiteit Leuven: www.kuleuven.be/Prometheus. The authors wish to thank Dr. Alf Gerisch for his assistance.

Reference List

1. Bailon-Plaza,A., andM.C.van der Meulen. 2001. A mathematical framework to study the effects of growth factor influences on fracture healing. J. Theor. Biol. 212:191-209.

2. Barnes,G.L., P.J.Kostenuik, L.C.Gerstenfeld, and T.A.Einhorn. 1999. Growth factor regulation of fracture repair. Journal of Bone and Mineral Research 14:1805-1815.

3. Bentley,K., H.Gerhardt, and P.A.Bates. 2008. Agent-based simulation of notch-mediated tip cell selection in angiogenic sprout initialisation. J. Theor. Biol. 250:25-36.

4. Bouletreau,P.J., S.M.Warren, J.A.Spector, Z.M.Peled, R.P.Gerrets, J.A.Greenwald, and M.T.Longaker. 2002. Hypoxia and VEGF up-regulate BMP-2 mRNA and protein expression in microvascular endothelial cells: Implications for fracture healing. Plastic and Reconstructive Surgery 109:2384-2397.

5. Brandl,A., A.Hartmann, V.Bechmann, B.Graf, M.Nerlich, and P.Angele. 2011. Oxidative Stress Induces Senescence in Chondrocytes. Journal of Orthopaedic Research 29:11141120.

6. Brighton,C.T., andA.G.Krebs. 1972. Oxygen-Tension of Healing Fractures in Rabbit. Journal of Bone and Joint Surgery-American Volume A 54:323-\&. 
7. Brighton,C.T., J.L.Schaffer, D.B.Shapiro, J.J.S.Tang, and C.C.Clark. 1991. Proliferation and Macromolecular-Synthesis by Rat Calvarial Bone-Cells Grown in Various OxygenTensions. Journal of Orthopaedic Research 9:847-854.

8. Brinker,M.R., andD.E.Bailey. 1997. Fracture healing in tibia fractures with an associated vascular injury. Journal of Trauma-Injury Infection and Critical Care 42:11-19.

9. Bruder,S.P., andB.S.Fox. 1999. Tissue engineering of bone - Cell based strategies. Clinical Orthopaedics and Related Research S68-S83.

10. Burke,D.P., andD.J.Kelly. 2012. Substrate Stiffness and Oxygen as Regulators of Stem Cell Differentiation during Skeletal Tissue Regeneration: A Mechanobiological Model. Plos One 7:e40737.

11. Cameron,J., D.Milner, J.Lee, J.Cheng, N.Fang, and I.Jasiuk. 2013. Employing the Biology of Successful Fracture Repair to Heal Critical Size Bone Defects. pp. 113-132. in: E.Heber-Katz, D.L.Stocum (Eds.), New Perspectives in Regeneration, Springer Berlin Heidelberg, Springer Berlin Heidelberg, pp. 113-132.

12. Carlier,A., L.Geris, K.Bentley, G.Carmeliet, P.Carmeliet, and O.H.Van. 2012. MOSAIC: a multiscale model of osteogenesis and sprouting angiogenesis with lateral inhibition of endothelial cells. PLoS Comput. Biol. 8:e1002724.

13. Chae,H.J., S.C.Kim, K.S.Han, S.W.Chae, N.H.An, H.M.Kim, H.H.Kim, Z.H.Lee, and H.R.Kim. 2001. Hypoxia induces apoptosis by caspase activation accompanying cytochrome $C$ release from mitochondria in MC3T3E1 osteoblasts. p38 MAPK is related in hypoxiainduced apoptosis. Immunopharmacology and Immunotoxicology 23:133-152.

14. Cheema,U., R.A.Brown, B.Alp, and A.J.MacRobert. 2008. Spatially defined oxygen gradients and vascular endothelial growth factor expression in an engineered 3D cell model. Cellular and Molecular Life Sciences 65:177-186.

15. Chen,G., F.Niemeyer, T.Wehner, U.Simon, M.A.Schuetz, M.J.Pearcy, and L.E.Claes. 2009. Simulation of the nutrient supply in fracture healing. J Biomech. 42:2575-2583.

16. Cho,T.J., L.C.Gerstenfeld, and T.A.Einhorn. 2002. Differential temporal expression of members of the transforming growth factor beta superfamily during murine fracture healing. Journal of Bone and Mineral Research 17:513-520.

17. Cochran,D.M., D.Fukumura, M.Ancukiewicz, P.Carmeliet, and R.K.Jain. 2006. Evolution of oxygen and glucose concentration profiles in a tissue-mimetic culture system of embryonic stem cells. Annals of Biomedical Engineering 34:1247-1258.

18. Colnot,C. 2009. Skeletal Cell Fate Decisions Within Periosteum and Bone Marrow During Bone Regeneration. Journal of Bone and Mineral Research 24:274-282.

19. De Bock,K., M.Georgiadou, S.Schoors, A.Kuchnio, B.W.Wong, A.R.Cantelmo, A.Quaegebeur, B.Ghesquiere, S.Cauwenberghs, G.Eelen, L.K.Phng, I.Betz, B.Tembuyser, K.Brepoels, J.Welti, I.Geudens, I.Segura, B.Cruys, F.Bifari, I.Decimo, R.Blanco, S.Wyns, J.Vangindertael, S.Rocha, R.T.Collins, S.Munck, D.Daelemans, H.Imamura, R.Devlieger, M.Rider, P.P.Van Veldhoven, F.Schuit, R.Bartrons, J.Hofkens, P.Fraisl, S.Telang, R.J.DeBerardinis, L.Schoonjans, S.Vinckier, J.Chesney, H.Gerhardt, 
M.Dewerchin, and P.Carmeliet. 2013. Role of PFKFB3-Driven Glycolysis in Vessel Sprouting. Cell 154:651-663.

20. Demol,J., D.Lambrechts, L.Geris, J.Schrooten, and O.H.Van. 2011. Towards a quantitative understanding of oxygen tension and cell density evolution in fibrin hydrogels. Biomaterials 32:107-118.

21. Deschepper,M., K.Oudina, B.David, V.Myrtil, C.Collet, M.Bensidhoum, D.Logeart-Avramoglou, and H.Petite. 2011. Survival and function of mesenchymal stem cells (MSCs) depend on glucose to overcome exposure to long-term, severe and continuous hypoxia. Journal of Cellular and Molecular Medicine 15:1505-1514.

22. Dimitriou,R., E.Jones, D.McGonagle, and P.V.Giannoudis. 2011. Bone regeneration: current concepts and future directions. BMC Med. 9:66.

23. Dimitriou,R., E.Tsiridis, and P.V.Giannoudis. 2005. Current concepts of molecular aspects of bone healing. Injury-International Journal of the Care of the Injured 36:1392-1404.

24. Drosse,I., E.Volkmer, S.Seitz, H.Seitz, R.Penzkofer, K.Zahn, U.Matis, W.Mutschler, P.Augat, and M.Schieker. 2008. Validation of a femoral critical size defect model for orthotopic evaluation of bone healing: A biomechanical, veterinary and trauma surgical perspective. Tissue Engineering Part C-Methods 14:79-88.

25. Einhorn,T.A. 1998. The cell and molecular biology of fracture healing. Clinical Orthopaedics and Related Research S7-S21.

26. Epari,D.R., J.Lienau, H.Schell, F.Witt, and G.N.Duda. 2008. Pressure, oxygen tension and temperature in the periosteal callus during bone healing - An in vivo study in sheep. Bone 43:734-739.

27. Fermor,B., J.Urban, D.Murray, A.Pocock, E.Lim, M.Francis, and J.Gage. 1998. Proliferation and collagen synthesis of human anterior cruciate ligament cells in vitro: Effects of ascorbate-2-phosphate, dexamethasone and oxygen tension. Cell Biology International 22:635-640.

28. Fraisl,P., M.Mazzone, T.Schmidt, and P.Carmeliet. 2009. Regulation of Angiogenesis by Oxygen and Metabolism. Developmental Cell 16:167-179.

29. Geris,L., A.Gerisch, J.V.Sloten, R.Weiner, and H.V.Oosterwyck. 2008. Angiogenesis in bone fracture healing: a bioregulatory model. J. Theor. Biol. 251:137-158.

30. Geris,L., A.A.Reed, S.J.Vander, A.H.Simpson, and O.H.Van. 2010. Occurrence and treatment of bone atrophic non-unions investigated by an integrative approach. PLoS. Comput. Biol. 6:e1000915.

31. Geris,L., S.J.Vander, and O.H.Van. 2009. In silico biology of bone modelling and remodelling: regeneration. Philos. Transact. A Math. Phys. Eng Sci. 367:2031-2053.

32. Gerisch,A., andM.A.J.Chaplain. 2006. Robust numerical methods for taxis-diffusion-reaction systems: Applications to biomedical problems. Mathematical and Computer Modelling 43:49-75. 
33. Gerstenfeld,L.C., D.M.Cullinane, G.L.Barnes, D.T.Graves, and T.A.Einhorn. 2003a. Fracture healing as a post-natal developmental process: Molecular, spatial, and temporal aspects of its regulation. Journal of Cellular Biochemistry 88:873-884.

34. Gerstenfeld,L.C., D.M.Cullinane, G.L.Barnes, D.T.Graves, and T.A.Einhorn. 2003b. Fracture healing as a post-natal developmental process: molecular, spatial, and temporal aspects of its regulation. J. Cell Biochem. 88:873-884.

35. Grayson,W.L., F.Zhao, B.Bunnell, and T.Ma. 2007. Hypoxia enhances proliferation and tissue formation of human mesenchymal stem cells. Biochem. Biophys. Res. Commun. 358:948-953.

36. Grimshaw,M.J., andR.M.Mason. 2000. Bovine articular chondrocyte function in vitro depends upon oxygen tension. Osteoarthritis and Cartilage 8:386-392.

37. Hansen-Algenstaedt,N., C.Joscheck, L.Wolfram, C.Schaefer, I.Muller, A.Bottcher, G.Deuretzbacher, L.Wiesner, M.Leunig, P.Algenstaedt, and W.Ruther. 2006. Sequential changes in vessel formation and microvascular function during bone repair. Acta Orthopaedica 77:429-439.

38. Harrison,L.J., J.L.Cunningham, L.Stromberg, and A.E.Goodship. 2003. Controlled induction of a pseudarthrosis: A study using a rodent model. Journal of Orthopaedic Trauma 17:11-21.

39. Harry,L.E., A.Sandison, M.F.Pearse, E.M.Paleolog, and J.Nanchahal. 2009. Comparison of the Vascularity of Fasciocutaneous Tissue and Muscle for Coverage of Open Tibial Fractures. Plastic and Reconstructive Surgery 124:1211-1219.

40. Henrotin,Y., B.Kurz, and T.Aigner. 2005. Oxygen and reactive oxygen species in cartilage degradation: friends or foes? Osteoarthritis and Cartilage 13:643-654.

41. Hirao,M., N.Tamai, N.Tsumaki, H.Yoshikawa, and A.Myoui. 2006. Oxygen tension regulates chondrocyte differentiation and function during endochondral ossification. Journal of Biological Chemistry 281:31079-31092.

42. Hojo,H., S.Ohba, K.Taniguchi, M.Shirai, F.Yano, T.Saito, T.Ikeda, K.Nakajima, Y.Komiyama, N.Nakagata, K.Suzuki, Y.Mishina, M.Yamada, T.Konno, T.Takato, H.Kawaguchi, H.Kambara, and U.Chung. 2013. Hedgehog-Gli Activators Direct Osteo-chondrogenic Function of Bone Morphogenetic Protein toward Osteogenesis in the Perichondrium. Journal of Biological Chemistry 288:9924-9932.

43. Holzwarth,C., M.Vaegler, F.Gieseke, S.M.Pfister, R.Handgretinger, G.Kerst, and I.Muller. 2010. Low physiologic oxygen tensions reduce proliferation and differentiation of human multipotent mesenchymal stromal cells. Bmc Cell Biology 11.

44. Isaksson,H. 2012. Recent advances in mechanobiological modeling of bone regeneration. Mechanics Research Communications 42:22-31.

45. Isaksson,H., W.Wilson, C.C.van Donkelaar, R.Huiskes, and K.Ito. 2006. Comparison of biophysical stimuli for mechano-regulation of tissue differentiation during fracture healing. Journal of Biomechanics 39:1507-1516. 
46. Ji,J.W., N.M.Tsoukias, D.Goldman, and A.S.Popel. 2006. A computational model of oxygen transport in skeletal muscle for sprouting and splitting modes of angiogenesis. Journal of Theoretical Biology 241:94-108.

47. Kanichai,M., D.Ferguson, P.J.Prendergast, and V.A.Campbell. 2008. Hypoxia promotes chondrogenesis in rat mesenchymal stem cells: A role for AKT and hypoxia-inducible factor (HIF)-1 alpha. Journal of Cellular Physiology 216:708-715.

48. Komarova,S.V., F.I.Ataullakhanov, and R.K.Globus. 2000. Bioenergetics and mitochondrial transmembrane potential during differentiation of cultured osteoblasts. American Journal of Physiology-Cell Physiology 279:C1220-C1229.

49. Komatsu,D.E., andM.Hadjiargyrou. 2004. Activation of the transcription factor HIF-1 and its target genes, VEGF, HO-1, iNOS, during fracture repair. Bone 34:680-688.

50. Kubo,S., G.M.Cooper, T.Matsumoto, J.A.Phillippi, K.A.Corsi, A.Usas, G.H.Li, F.H.Fu, and J.Huard. 2009. Blocking Vascular Endothelial Growth Factor With Soluble Flt-1 Improves the Chondrogenic Potential of Mouse Skeletal Muscle-Derived Stem Cells. Arthritis and Rheumatism 60:155-165.

51. Kwon,S.H., T.J.Lee, J.Park, J.E.Hwang, M.Jin, H.K.Jang, N.S.Hwang, and B.S.Kim. 2013. Modulation of BMP-2-Induced Chondrogenic Versus Osteogenic Differentiation of Human Mesenchymal Stem Cells by Cell-Specific Extracellular Matrices. Tissue Engineering Part A 19:49-58.

52. Lacroix,D., andP.J.Prendergast. 2002. A mechano-regulation model for tissue differentiation during fracture healing: analysis of gap size and loading. J. Biomech. 35:1163-1171.

53. Lennon,D.P., J.M.Edmison, and A.I.Caplan. 2001. Cultivation of rat marrow-derived mesenchymal stem cells in reduced oxygen tension: effects on in vitro and in vivo osteochondrogenesis. J. Cell Physiol 187:345-355.

54. Lissenberg-Thunnissen,S.N., D.J.de Gorter, C.F.Sier, and I.B.Schipper. 2011. Use and efficacy of bone morphogenetic proteins in fracture healing. Int. Orthop. 35:1271-1280.

55. Liu,G., A.A.Qutub, P.Vempati, F.Mac Gabhann, and A.S.Popel. 2011. Module-based multiscale simulation of angiogenesis in skeletal muscle. Theoretical Biology and Medical Modelling 8.

56. Lu,C.Y., T.Miclau, D.Hu, and R.S.Marcucio. 2007. Ischemia leads to delayed union during fracture healing: A mouse model. Journal of Orthopaedic Research 25:51-61.

57. Lu,C.Y., N.Saless, X.D.Wang, A.Sinha, S.Decker, G.Kazakia, H.G.Hou, B.Williams, H.M.Swartz, T.K.Hunt, T.Miclau, and R.S.Marcucio. 2013a. The role of oxygen during fracture healing. Bone 52:220-229.

58. Lu,C.Y., N.Saless, X.D.Wang, A.Sinha, S.Decker, G.Kazakia, H.G.Hou, B.Williams, H.M.Swartz, T.K.Hunt, T.Miclau, and R.S.Marcucio. 2013b. The role of oxygen during fracture healing. Bone 52:220-229.

59. MACDOUGA.JD, andM.Mccabe. 1967. Diffusion Coefficient of Oxygen Through Tissues. Nature 215:1173-\&. 
60. Maes,C., G.Carmeliet, and E.Schipani. 2012. Hypoxia-driven pathways in bone development, regeneration and disease. Nature Reviews Rheumatology 8:358-366.

61. Malda,J., J.Rouwkema, D.E.Martens, E.P.le Comte, F.K.Kooy, J.Tramper, C.A.van Blitterswijk, and J.Riesle. 2004a. Oxygen gradients in tissue-engineered PEGT/PBT cartilaginous constructs: Measurement and modeling. Biotechnology and Bioengineering 86:9-18.

62. Malda,J., J.Rouwkema, D.E.Martens, E.P.le Comte, F.K.Kooy, J.Tramper, C.A.van Blitterswijk, and J.Riesle. 2004b. Oxygen gradients in tissue-engineered PEGT/PBT cartilaginous constructs: Measurement and modeling. Biotechnology and Bioengineering 86:9-18.

63. Malladi,P., Y.Xu, M.Chiou, A.J.Giaccia, and M.T.Longaker. 2006. Effect of reduced oxygen tension on chondrogenesis and osteogenesis in adipose-derived mesenchymal cells. American Journal of Physiology-Cell Physiology 290:C1139-C1145.

64. Marsh,D. 1998. Concepts of fracture union, delayed union, and nonunion. Clin. Orthop. Relat Res. S22-S30.

65. Masquelet,A.C. 2003. Muscle reconstruction in reconstructive surgery: soft tissue repair and long bone reconstruction. Langenbecks Archives of Surgery 388:344-346.

66. McDougall,S.R., A.R.A.Anderson, M.A.J.Chaplain, and J.A.Sherratt. 2002. Mathematical modelling of flow through vascular networks: Implications for ibmour-induced angiogenesis and chemotherapy strategies. Bulletin of Mathematical Biology 64:673702.

67. Merceron,C., C.Vinatier, S.Portron, M.Masson, J.Amiaud, L.Guigand, Y.Cherel, P.Weiss, and J.Guicheux. 2010. Differential effects of hypoxia on osteochondrogenic potential of human adipose-derived stem cells. American Journal of Physiology-Cell Physiology 298:C355-C364.

68. Mertens,S., T.Noll, R.Spahr, A.Krutzfeldt, and H.M.Piper. 1990. Energetic Response of Coronary Endothelial-Cells to Hypoxia. American Journal of Physiology 258:H689H694.

69. Meyer,E.G., C.T.Buckley, S.D.Thorpe, and D.J.Kelly. 2010. Low oxygen tension is a more potent promoter of chondrogenic differentiation than dynamic compression. Journal of Biomechanics 43:2516-2523.

70. Murao,H., K.Yamamoto, S.Matsuda, and H.Akiyama. 2013. Periosteal cells are a major source of soft callus in bone fracture. J Bone Miner Metab 1-9.

71. Nicolaije,C., M.Koedam, and J.P.van Leeuwen. 2012. Decreased oxygen tension lowers reactive oxygen species and apoptosis and inhibits osteoblast matrix mineralization through changes in early osteoblast differentiation. J. Cell Physiol 227:1309-1318.

72. Papandreou,I., R.A.Cairns, L.Fontana, A.L.Lim, and N.C.Denko. 2006. HIF-1 mediates adaptation to hypoxia by actively downregulating mitochondrial oxygen consumption. Cell Metabolism 3:187-197.

73. Patterson,T.E., K.Kumagai, L.Griffith, and G.F.Muschler. 2008. Cellular strategies for enhancement of fracture repair. Journal of Bone and Joint Surgery-American Volume 90A:111-119. 
74. Peiffer,V., A.Gerisch, D.Vandepitte, O.H.Van, and L.Geris. 2011. A hybrid bioregulatory model of angiogenesis during bone fracture healing. Biomech. Model. Mechanobiol. 10:383395.

75. Peters,K., G.Kamp, A.Berz, R.E.Unger, S.Barth, A.Salamon, J.Rychly, and C.J.Kirkpatrick. 2009. Changes in Human Endothelial Cell Energy Metabolic Capacities during in vitro Cultivation. The Role of "Aerobic Glycolysis" and Proliferation. Cellular Physiology and Biochemistry 24:483-492.

76. Pivonka,P., andC.R.Dunstan. 2012. Role of mathematical modeling in bone fracture healing. BoneKEy Rep 1.

77. Potier,E., E.Ferreira, A.Meunier, L.Sedel, D.Logeart-Avramoglou, and H.Petite. 2007. Prolonged hypoxia concomitant with serum deprivation induces massive human mesenchymal stem cell death. Tissue Engineering 13:1325-1331.

78. Prendergast,P.J., R.Huiskes, and K.Soballe. 1997. Biophysical stimuli on cells during tissue differentiation at implant interfaces. Journal of Biomechanics 30:539-548.

79. Pugh,C.W., andP.J.Ratcliffe. 2003. Regulation of angiogenesis by hypoxia: role of the HIF system. Nature Medicine 9:677-684.

80. Qutub,A.A., andA.S.Popel. 2009. Elongation, proliferation \& migration differentiate endothelial cell phenotypes and determine capillary sprouting. Bmc Systems Biology 3.

81. Ren,H.Y., Y.Cao, Q.J.Zhao, J.Li, C.X.Zhou, L.M.Liao, M.Y.Jia, Q.Zhao, H.G.Cai, Z.C.Han, R.C.Yang, G.Q.Chen, and R.C.H.Zhao. 2006. Proliferation and differentiation of bone marrow stromal cells under hypoxic conditions. Biochemical and Biophysical Research Communications 347:12-21.

82. Roberts,T.T., andA.J.Rosenbaum. 2012. Bone grafts, bone substitutes and orthobiologics The bridge between basic science and clinical advancements in fracture healing. Organogenesis 8:114-124.

83. Rumsey,W.L., C.Schlosser, E.M.Nuutinen, M.Robiolio, and D.F.Wilson. 1990. Cellular Energetics and the Oxygen Dependence of Respiration in Cardiac Myocytes Isolated from Adult-Rat. Journal of Biological Chemistry 265:15392-15399.

84. Simon,U., P.Augat, M.Utz, and L.Claes. 2011. A numerical model of the fracture healing process that describes tissue development and revascularisation. Comput. Methods Biomech. Biomed. Engin. 14:79-93.

85. Stevens,M.M. 2008. Biomaterials for bone tissue engineering. Materials Today 11:18-25.

86. Street,J., M.Bao, L.deGuzman, S.Bunting, F.V.Peale, N.Ferrara, H.Steinmetz, J.Hoeffel, J.L.Cleland, A.Daugherty, N.van Bruggen, H.P.Redmond, R.A.D.Carano, and E.H.Filvaroff. 2002. Vascular endothelial growth factor stimulates bone repair by promoting angiogenesis and bone turnover. Proceedings of the National Academy of Sciences of the United States of America 99:9656-9661. 
87. Taguchi,K., R.Ogawa, M.Migita, H.Hanawa, H.Ito, and H.Orimo. 2005. The role of bone marrow-derived cells in bone fracture repair in a green fluorescent protein chimeric mouse model. Biochemical and Biophysical Research Communications 331:31-36.

88. Tolli,H., S.Kujala, T.Jamsa, and P.Jalovaara. 2011. Reindeer bone extract can heal the criticalsize rat femur defect. International Orthopaedics 35:615-622.

89. Vogelin,E., N.F.Jones, J.I.Huang, J.H.Brekke, and J.R.Lieberman. 2005. Healing of a criticalsized defect in the rat femur with use of a vascularized periosteal flap, a biodegradable matrix, and bone morphogenetic protein. Journal of Bone and Joint Surgery-American Volume 87A:1323-1331.

90. Wagegg,M., T.Gaber, F.L.Lohanatha, M.Hahne, C.Strehl, M.Fangradt, C.L.Tran, K.Schonbeck, P.Hoff, A.Ode, C.Perka, G.N.Duda, and F.Buttgereit. 2012. Hypoxia Promotes Osteogenesis but Suppresses Adipogenesis of Human Mesenchymal Stromal Cells in a Hypoxia-Inducible Factor-1 Dependent Manner. Plos One 7.

91. Wan,C., S.R.Gilbert, Y.Wang, X.Cao, X.Shen, G.Ramaswamy, K.A.Jacobsen, Z.S.Alaql, A.W.Eberhardt, L.C.Gerstenfeld, T.A.Einhorn, L.Deng, and T.L.Clemens. 2008. Activation of the hypoxia-inducible factor-1 alpha pathway accelerates bone regeneration. Proceedings of the National Academy of Sciences of the United States of America 105:686-691.

92. Weiner,R., B.A.Schmitt, and H.Podhaisky. 1997. ROWMAP - a ROW-code with Krylov techniques for large stiff ODEs. Applied Numerical Mathematics 25:303-319.

93. Xie,C., B.J.Liang, M.Xue, A.S.P.Lin, A.Loiselle, E.M.Schwarz, R.E.Guldberg, R.J.O'Keefe, and X.P.Zhang. 2009. Rescue of Impaired Fracture Healing in COX-2(-/-) Mice via Activation of Prostaglandin E2 Receptor Subtype 4. American Journal of Pathology 175:772-785.

94. Xu,Y., P.Malladi, M.Chiou, E.Bekerman, A.J.Giaccia, and M.T.Longaker. 2007. In vitro expansion of adipose-derived adult stromal cells in hypoxia enhances early chondrogenesis. Tissue Engineering 13:2981-2993.

95. Zelzer,E., D.J.Glotzer, C.Hartmann, D.Thomas, N.Fukai, S.Soker, and B.R.Olsen. 2001. Tissue specific regulation of VEGF expression during bone development requires Cbfa1/Runx2. Mechanisms of Development 106:97-106.

96. Zscharnack,M., C.Poesel, J.Galle, and A.Bader. 2009. Low Oxygen Expansion Improves Subsequent Chondrogenesis of Ovine Bone-Marrow-Derived Mesenchymal Stem Cells in Collagen Type I Hydrogel. Cells Tissues Organs 190:81-93. 